\title{
Accessory parameters in confluent Heun equations and classical irregular conformal blocks
}

\author{
O. Lisovyy ${ }^{17}$ A. Naidiuk ${ }^{2}$ \\ ${ }^{a}$ Institut Denis-Poisson, Université de Tours, CNRS, Parc de Grandmont, 37200 Tours, France
}

\begin{abstract}
Classical Virasoro conformal blocks are believed to be directly related to accessory parameters of Floquet type in the Heun equation and some of its confluent versions. We extend this relation to another class of accessory parameter functions that are defined by inverting all-order Bohr-Sommerfeld periods for confluent and biconfluent Heun equation. The relevant conformal blocks involve Nagoya irregular vertex operators of rank 1 and 2 and conjecturally correspond to partition functions of a $4 \mathrm{D} \mathscr{N}=2, N_{f}=3$ gauge theory at strong coupling and an Argyres-Douglas theory.
\end{abstract}

\section{Introduction and summary of results}

\subsection{Heun accessory parameter and classical conformal blocks}

The Heun's differential equation (HE), written in normal form, is given by

$$
\begin{gathered}
\psi^{\prime \prime}(z)=V(z) \psi(z), \\
V(z)=\frac{\theta_{0}^{2}-\frac{1}{4}}{z^{2}}+\frac{\theta_{1}^{2}-\frac{1}{4}}{(z-1)^{2}}+\frac{\theta_{t}^{2}-\frac{1}{4}}{(z-t)^{2}}+\frac{\theta_{\infty}^{2}-\theta_{0}^{2}-\theta_{1}^{2}-\theta_{t}^{2}+\frac{1}{2}}{z(z-1)}+\frac{(1-t) \mathscr{E}}{z(z-1)(z-t)} .
\end{gathered}
$$

Every 2nd order ODE with at most 4 regular singular points on the Riemann sphere can be reduced to 1.1 by a Möbius transformation mapping these points to $0, t, 1, \infty$. The exponents of local monodromy are encoded into the Riemann scheme

\begin{tabular}{c|c|c|c}
0 & 1 & $\mathrm{t}$ & $\infty$ \\
\hline$\frac{1}{2} \pm \theta_{0}$ & $\frac{1}{2} \pm \theta_{1}$ & $\frac{1}{2} \pm \theta_{t}$ & $\frac{1}{2} \pm \theta_{\infty}$
\end{tabular}

The main new feature of [1.1, as compared to the case of 3 regular singularities described by the Gauss hypergeometric equation, is the presence of an accessory parameter $\mathscr{E}$, which does not influence the local behavior of solutions. This makes the global analysis of Heun's equation considerably more difficult, the central question being the dependence of $\mathscr{E}$ on monodromy.

Indeed, the Riemann-Hilbert correspondence assigns to every choice of $\mathscr{E}$ and $t$ a point in the space of monodromy data which consists of conjugacy classes of 4 -tuples of SL $(2, \mathbb{C})$-matrices with fixed spectrum that multiply to identity:

$$
\mathscr{M}=\left\{M_{0}, M_{1}, M_{t}, M_{\infty} \in \operatorname{SL}(2, \mathbb{C}): M_{\infty} M_{1} M_{t} M_{0}=\mathbf{1}, \operatorname{Tr} M_{k}=-2 \cos 2 \pi \theta_{k} \text { for } k=0,1, t, \infty\right\} / \sim .
$$

The space $\mathscr{M}$ is generically 2-dimensional and $(\mathscr{E}, t)$ can therefore be regarded as a pair of local coordinates on it. Any other convenient coordinate $\xi$ on $\mathscr{M}$ may be viewed as a function of $(\mathscr{E}, t)$. Inverting this relation, to any choice of $\xi$ we may assign an accessory parameter function $\mathscr{E}^{[\xi]}(t \mid \xi)$, where the upper index is introduced to emphasize that $\mathscr{E}^{[\xi]}$ depends not only on the value of $\xi$ but also on how this coordinate was chosen. A standard choice is to take as $\xi$ one of the three Floquet exponents $\sigma_{k t}$ defined by

$$
\operatorname{Tr} M_{k} M_{t}=-2 \cos 2 \pi \sigma_{k t}, \quad k=0,1, \infty .
$$

We thereby obtain three different accessory parameter functions - however, since different singular points of 1.1 can be exchanged by Möbius transformations, these functions turn out to be related by a permutation of parameters $\vec{\theta}=\left(\theta_{0}, \theta_{1}, \theta_{t}, \theta_{\infty}\right)$ combined with an appropriate transformation of $t$.

\footnotetext{
${ }^{1}$ lisovyi@lmpt.univ-tours.fr

2 andrii.naidiuk@lmpt.univ-tours.fr
} 
The direct monodromy problem for Heun's equation consists essentially in finding $\sigma_{k t}$ 's for given $(\mathscr{E}, t)$; it can be formally solved using Hill determinants. Remarkably, the inverse monodromy problem of reconstruction of Heun's equation with prescribed monodromy exponent $\sigma_{k t}$ can be solved more efficiently. The result is given by a perturbative series such as

$$
\mathscr{E}^{\left[\sigma_{0 t}\right]}(t \mid \sigma)=\sum_{n=0}^{\infty} \mathscr{E}_{n}^{\left[\sigma_{0 t}\right]}(\sigma) t^{n}
$$

whose coefficients $\mathscr{E}_{k}^{\left[\sigma_{0 t}\right]}$ can be found recursively using continued fractions. Both Hill determinant and continued fraction method are well-known since the end of 19th century in the context of Mathieu equation, see e.g. [WW Chapter 19], [AS Chapter 20], [Hei], [DLMF, Eq.28.15.E1]. The Mathieu counterparts of functions $\sigma_{0 t}(\mathscr{E}, t)$ and $\mathscr{E}^{\left[\sigma_{0 t}\right]}(t \mid \sigma)$ are implemented in Mathematica as MathieuCharacteristicExponent and MathieuCharacteristicA. Although extension of continued fractions to Heun is straightforward, it does not seem to be widely known and is included to Appendix A

The second class of special functions studied in this work are Virasoro conformal blocks (CBs). The regular 4-point spherical $s$-channel CB,

$$
\mathscr{F}\left(t \mid c,\left\{\Delta_{k}\right\}\right)=t^{\Delta_{\sigma}-\Delta_{0}-\Delta_{t}}\left[1+\sum_{n=1}^{\infty} \mathscr{F}_{n}\left(c,\left\{\Delta_{k}\right\}\right) t^{n}\right] .
$$

depends on the anharmonic ratio $t$ and six complex parameters: the Virasoro central charge $c$ and five conformal dimensions $\Delta_{0,1, t, \infty, \sigma}$. The coefficients $\mathscr{F}_{n}$ are rational in $c,\left\{\Delta_{k}\right\}$ and are fixed by the commutation relations of the Virasoro algebra [BPZ. Their explicit form can also be obtained using the AGT correspondence [AGT identifying $\mathscr{F}\left(t \mid c,\left\{\Delta_{k}\right\}\right)$ with Nekrasov partition function Nek1 NO] of $\mathscr{N}=24 \mathrm{D}$ supersymmetric SU (2) gauge theory with $N_{f}=4$ flavors.

The relation between Heun accessory parameter functions and regular CBs is given by a composition of two conjectures formulated in 1986 paper of Zamolodchikov [Zam, Eqs. (2.30)-(2.32) $]^{3}$

- Conjecture A (exponentiation). It states that in the quasiclassical limit

$$
c, \Delta_{0,1, t, \infty, \sigma} \rightarrow \infty, \quad \frac{6 \Delta_{k}}{c} \rightarrow \delta_{k}, \quad k=0,1, t, \infty, \sigma
$$

conformal block [1.5] has WKB type asymptotics

$$
\mathscr{F}\left(t \mid\left\{\Delta_{k}\right\}\right) \sim \exp \frac{c}{6} \mathscr{W}\left(t \mid\left\{\delta_{k}\right\}\right) .
$$

The series

$$
\mathscr{W}\left(t \mid\left\{\delta_{k}\right\}\right)=\left(\delta_{\sigma}-\delta_{0}-\delta_{t}\right) \ln t+\sum_{n=1}^{\infty} \mathscr{W}_{n}\left(\left\{\delta_{k}\right\}\right) t^{n}
$$

appearing in this asymptotics is called classical conformal block.

- Conjecture B (Heun/classical CB correspondence). Classical CB and Heun accessory parameter function are related by

$$
\mathscr{E}^{\left[\sigma_{0 t}\right]}(t \mid \sigma, \vec{\theta})=t \frac{\partial}{\partial t} \mathscr{W}\left(t \mid\left\{\delta_{k}\right\}\right)
$$

where the rescaled conformal dimensions are identified with Heun monodromy exponents via

$$
\delta_{\sigma}=\frac{1}{4}-\sigma^{2}, \quad \delta_{k}=\frac{1}{4}-\theta_{k}^{2}, \quad k=0,1, t, \infty
$$

Conjecture A is supported by semiclassical arguments in Liouville QFT and on the gauge theory side of the AGT correspondence where $\mathbb{W}(t)$ is interpreted as Nekrasov-Shatashvili effective twisted superpotential [NS]. The strongest evidence comes from the explicit computation of large-order CB coefficients. We also mention a recent paper $[\mathrm{BDK}]$ which claims to prove the exponentiation of the 4-point $\mathrm{CB}$ [1.5]. Conjecture B follows from a refined version of the exponentiation (the so-called heavy-light factorization) for conformal blocks with degenerate fields combined with BPZ decoupling equations [Tes, LLNZ] (see also [PP3 for details). It can also be tested directly by comparing the classical CB series (1.8) order by order with the accessory parameter expansion computed from continued fractions or by other approaches $[\mathrm{NC}, \mathrm{LN}, \mathrm{HK}, \mathrm{Men}$.

\footnotetext{
3 Zam further refers to BPZ; however, the discussion of the quasiclassical limit in BPZ is limited to a footnote on p. 357 which does not contain a neat formulation of any of the two hypotheses.
} 


\subsection{Confluent Heun equations}

Our aim is to extend Conjectures A and B to the irregular setting. Indeed, there is a number of confluent versions of the Heun's equation $[\mathrm{SL}]$ which we organize into a geometric degeneration diagram:

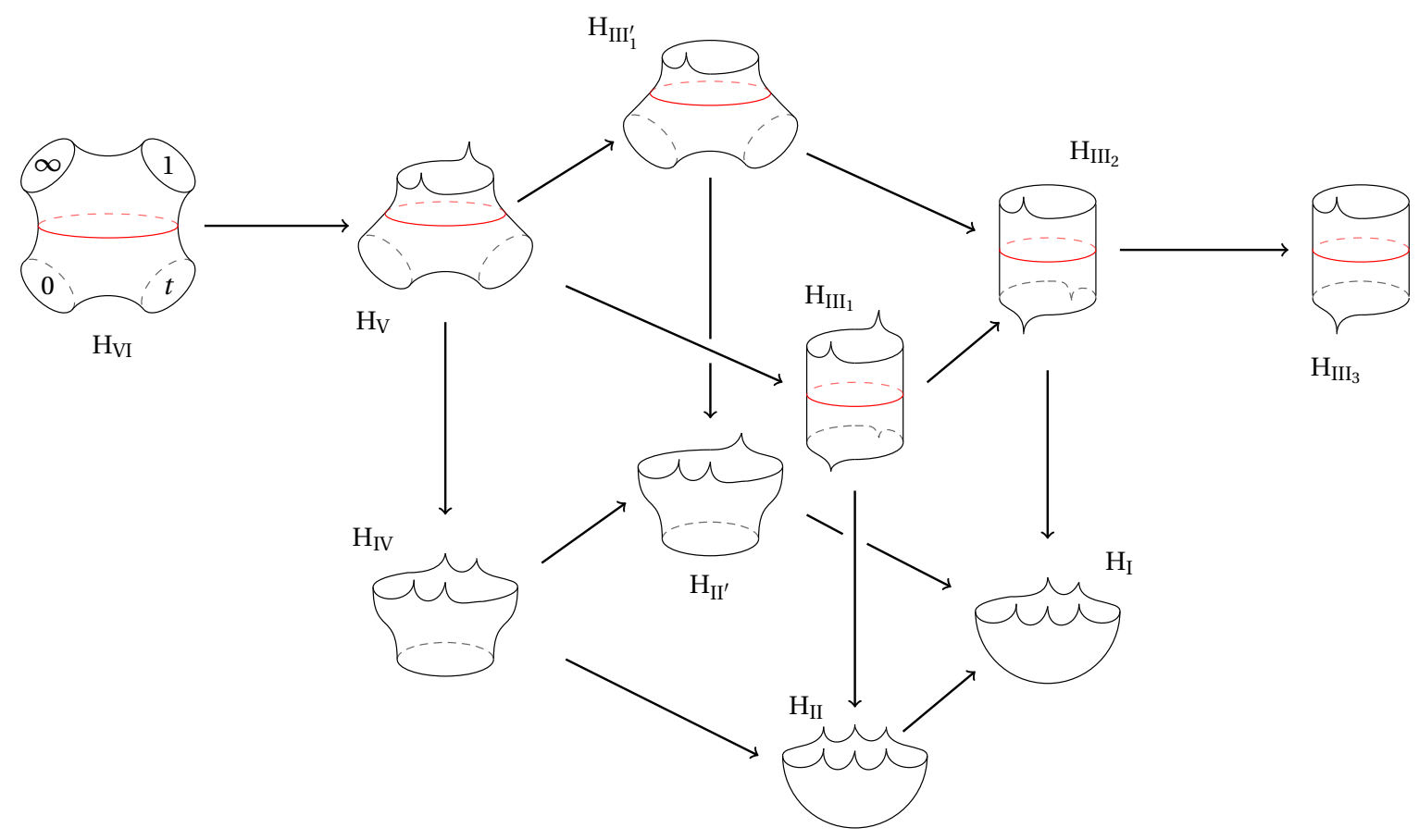

Each entry of this diagram represents an ODE of the form $\psi^{\prime \prime}(z)=V(z) \psi(z)$ on $\mathbb{C P}^{1}$. The form of the potential $V(z)$ can be read off from the corresponding Riemann surface. Every hole corresponds to a pole of the quadratic differential $V(z) d z^{2}$ whose order is equal to the number of cusps plus 2, so that non-cusped hole corresponds to a regular singular point. Thus $\mathrm{H}_{\mathrm{VI}}$ is the usual $\mathrm{HE}, \mathrm{H}_{\mathrm{V}}$ has two regular singular points and an irregular one, etc. We record the potentials in the following table:

\begin{tabular}{c|c|c} 
notation & equation name & potential $V(z)$ \\
\hline \hline $\mathrm{H}_{\mathrm{V}}$ & confluent HE & $\frac{\theta_{0}^{2}-\frac{1}{4}}{z^{2}}+\frac{\theta_{t}^{2}-\frac{1}{4}}{(z-t)^{2}}+\frac{1}{4}+\frac{\theta_{*}}{z}-\frac{\mathscr{E}}{z(z-t)}$ \\
\hline $\mathrm{H}_{\mathrm{IV}}$ & biconfluent HE & $\frac{\theta_{0}^{2}-\frac{1}{4}}{z^{2}}-\frac{\mathscr{E}}{z}+2 \theta \cdot+(z+t)^{2}$ \\
\hline $\mathrm{H}_{\mathrm{III}}$ & doubly confluent HE & $\frac{t^{2}}{4 z^{4}}+\frac{t \theta_{\star}}{z^{3}}-\frac{\mathscr{E}}{z^{2}}+\frac{\theta_{*}}{z}+\frac{1}{4}$ \\
\hline $\mathrm{H}_{\mathrm{III}}$ & reduced doubly confluent HE & $\frac{t}{z^{3}}-\frac{\mathscr{E}}{z^{2}}+\frac{\theta_{*}}{z}+\frac{1}{4}$ \\
\hline $\mathrm{H}_{\mathrm{III}}$ & doubly reduced doubly confluent HE & $\frac{t}{z^{3}}-\frac{\mathscr{E}}{z^{2}}+\frac{1}{z}$ \\
\hline $\mathrm{H}_{\mathrm{II}}$ & triconfluent HE & $\left(z^{2}+t\right)^{2}+2 \theta_{\circ} z+\mathscr{E}$ \\
\hline $\mathrm{H}_{\mathrm{I}}$ & reduced triconfluent HE & $4 z^{3}+2 t z+\mathscr{E}$ \\
\hline \hline $\mathrm{H}_{\mathrm{III}_{1}^{\prime}}$ & reduced confluent HE & $\frac{\theta_{0}^{2}-\frac{1}{4}}{z^{2}}+\frac{\theta_{t}^{2}-\frac{1}{4}}{(z-t)^{2}}+\frac{1}{z}-\frac{\mathscr{E}}{z(z-t)}$ \\
\hline $\mathrm{H}_{\mathrm{II}^{\prime}}$ & reduced biconfluent HE & $\frac{\theta_{0}^{2}-\frac{1}{4}}{z^{2}}+\frac{\mathscr{E}}{z}+t+z$
\end{tabular}

Table 1: Notation for confluent Heun equations 
The parameters such as $\theta_{0}, \theta_{t}, \theta_{*}, \theta_{\bullet}, \theta_{\star}, \theta_{\circ}$ are exponents of local monodromy around regular singular points or formal monodromy around the irregular ones; $\mathscr{E}$ denotes the accessory parameter. The "time" (or "coupling") $t$ parameterizes the exponential behavior of $\psi(z)$ at irregular singularities, except in the case of $\mathrm{H}_{\mathrm{V}}$ and $\mathrm{H}_{\mathrm{III}_{1}^{\prime}}$, where it can be equivalently chosen as the position of one of the 2 regular singular points.

A Laplace transform of $\mathrm{H}_{\mathrm{III}}$ and $\mathrm{H}_{\mathrm{II}}$ (applied to their canonical forms) transforms them into $\mathrm{H}_{\mathrm{III}_{1}^{\prime}}$ and $\mathrm{H}_{\mathrm{II}^{\prime}}$. The above scheme thus contains seven inequivalent confluent HEs. It is not a surprise that the geometric confluence diagram exactly reproduces the one suggested in $[\mathrm{CM}, \mathrm{CMR}$ for Lax pairs of Painlevé equations. Indeed, there exists a connection between Heun and Painlevé functions [FIKN Section 2.1.3 and Chapter 15], Nov going back to classical papers of Fuchs [Fuchs and Garnier [Gar which was also the subject of one of the last works of Boris Dubrovin [DK].

Confluent HEs appear in a wide range of physical applications. To mention a few examples, $\mathrm{H}_{\mathrm{I}}$ and $\mathrm{H}_{\mathrm{II}}$ are Schroedinger equations for cubic and quartic oscillator; $\mathrm{H}_{\mathrm{III}_{3}}$ is equivalent to the Mathieu equation (quantum pendulum) through the change of variables $f(x)=e^{-i x} \psi\left(\sqrt{t} e^{2 i x}\right) ; \mathrm{H}_{\mathrm{V}}$ arises in the black hole scattering [BPM] Lea, STU, Fiz, CCN] and Rabi model of quantum optics [MPS, ZXBL $[$ CCR], etc.

\subsection{Conjectures A and B: confluent case}

There already exist a few irregular extensions of Conjectures A and B. Immediately after the AGT discovery of $4 \mathrm{D} / 2 \mathrm{D}$ duality it was realized that Nekrasov partition functions of $\mathscr{N}=2$ SUSY gauge theories with lower number of flavors $\left(N_{f}=0,1,2,3\right)$ correspond to a class of irregular CBs; we will call them confluent CBs of the 1st kind. The algebraic construction of these CBs Gai, BMT, GT involves rank 1 Whittaker modules for the Virasoro algebra. They are given by weak coupling $(t \rightarrow 0)$ expansions similar to 1.5 whose coefficients are nothing but suitable limits of $\mathscr{F}_{n}\left(c,\left\{\Delta_{k}\right\}\right)$. The same applies to quasiclassical limit where confluent CBs of the 1st kind are expected to exponentiate [PP1, RZ1, RZ2, PP2] and produce Nekrasov-Shatashvili twisted superpotentials of the relevant gauge theories.

On the other hand, for confluent equations $\mathrm{H}_{\mathrm{V}}, \mathrm{H}_{\mathrm{III}_{1}}, \mathrm{H}_{\mathrm{III}_{2}}, \mathrm{H}_{\mathrm{III}_{3}}$ there exists a natural counterpart of the accessory parameter function $\mathscr{E}^{\left[\sigma_{0 t}\right]}(t \mid \sigma)$. Recall that the latter is fixed by the choice of monodromy parameter $\sigma_{0 t}$, which in the confluent cases should be replaced by the Floquet exponent of (non-formal!) monodromy around irregular singular point at $\infty$. The relevant cycles are shown in red color in the degeneration diagram. Slightly abusing the notation, we will denote the corresponding confluent accessory parameter function by $\mathscr{E}^{[\mathrm{F}]}(t \mid \sigma)$ and call it Floquet characteristic. Its small $t$ expansion can be computed using continued fractions exactly as in the Heun case (see Appendix B. We also have

$$
\begin{aligned}
& \mathscr{E}_{\mathrm{V}}^{[\mathrm{F}]}(t \mid \sigma)=\left.\lim _{\Lambda \rightarrow \infty} \mathscr{E}_{\mathrm{VI}}^{[\mathrm{FF}}\left(\frac{t}{\Lambda} \mid \sigma\right)\right|_{\theta_{1}=\frac{\Lambda+\theta_{*}}{2}, \theta_{\infty}=\frac{\Lambda-\theta_{*}}{2}}, \\
& \mathscr{E}_{\mathrm{III}}^{[\mathrm{F}]}(t \mid \sigma)=\lim _{\Lambda \rightarrow \infty}\left[\mathscr{E}_{\mathrm{V}}^{[\mathrm{F}]}\left(\frac{t}{\Lambda} \mid \sigma\right)-\theta_{0}^{2}-\theta_{t}^{2}+\frac{1}{2}\right]_{\theta_{0}=\frac{\Lambda-\theta_{\star}}{2}, \theta_{t}=\frac{\Lambda+\theta_{\star}}{2}} \\
& \mathscr{E}_{\mathrm{III}_{2}}^{[\mathrm{F}]}(t \mid \sigma)=\lim _{\theta_{\star} \rightarrow \infty} \mathscr{E}_{\mathrm{III}_{1}}^{[\mathrm{F}]}\left(t / \theta_{\star} \mid \sigma\right), \\
& \mathscr{E}_{\mathrm{III}_{3}}^{[\mathrm{F}]}(t \mid \sigma)=\lim _{\theta_{*} \rightarrow \infty} \mathscr{E}_{\mathrm{III}_{2}}^{[\mathrm{F}]}\left(t / \theta_{*} \mid \sigma\right) .
\end{aligned}
$$

A relation between the Floquet characteristic $\mathscr{E}_{\mathrm{III}_{3}}^{[\mathrm{F}]}(t \mid \sigma)$ (i.e. accessory parameter in the Mathieu equation) with Nekrasov-Shatashvili twisted superpotential $\mathscr{W}_{N_{f}=0}(t)$ of the pure gauge theory has appeared in [NS as the simplest example of Bethe/gauge correspondence. Using the AGT relation, this correspondence was reformulated in terms of classical irregular CBs in [PP1]. Further extensions to $N_{f}=1,2$ in [PP2, RZ1] and $N_{f}=3$ in CCC similarly involve Floquet characteristics of confluent equations $\mathrm{H}_{\mathrm{III}_{2}}, \mathrm{H}_{\mathrm{III}_{1}}$ and $\mathrm{H}_{\mathrm{V}}$. In all four cases, the analog of Conjecture B has the same form as [1.9):

$$
\mathscr{E}^{[\mathrm{F}]}(t \mid \sigma)=t \frac{\partial}{\partial t} \mathbb{W}_{N_{f}}(t)
$$

These developments are briefly reviewed in Subsection 2.2 and Appendix $B$ for convenience of the readers with little prior exposition to $4 \mathrm{D} / 2 \mathrm{D}$ vocabulary, and also to provide general statements in a uniform notation ${ }^{4}$

\footnotetext{
${ }^{4}$ For $N_{f}=2$, Refs. PP2 RZ1 deal (on the differential equations side) with the Whittaker-Hill equation which is a special case of $\mathrm{H}_{\mathrm{III}}$. Ref. [CCC uses gauge theory quantities to define the analog of the right side of [1.12] for $N_{f}=3$.
} 
The main goal of the present work is to initiate the study of confluent counterparts of Conjectures A and $\mathrm{B}$ in the strong coupling $(t \rightarrow \infty)$ regime. In this setting, it is much less clear what one should expect at both sides of the putative analog of the relation (1.9). First, one needs to introduce a new basis in the space of irregular CBs to describe the confluent limit of the regular CBs in the $u$-channel. Several candidates for these confluent CBs of the 2nd kind were suggested in the work of Gaiotto and Teschner [GT] which defines a collision limit of the regular $u$-channel CBs leading to finite answers. An algebraic definition of such CBs was proposed by Nagoya in [Na1, Na2]; its main new feature is an irregular vertex operator acting between two VirasoroWhittaker modules.

Note that the series for confluent CBs of the 2nd kind cannot be expected to converge. Instead, they should be considered as formal series in $\frac{1}{t}$ which represent asymptotic expansions of an actual function inside some sectors (or even only along some directions) at $\infty$. Different sectors/directions can in principle lead to different bases of confluent CBs of the 2nd kind and it may well turn out that the constructions of [GT, Na1, Na2] do not cover all of them. In any case, it is straightforward to test the quasiclassical asymptotics for confluent CBs of the 2nd kind that are already available. We address this question in Subsection 2.3by checking exponentiation (Conjecture A) in two cases: (a) 3-point CB with 2 regular punctures and 1 irregular puncture of rank 1 (b) 2-point CB with 1 regular and 1 irregular puncture of rank 2.

Trying to define the accessory parameter side of the confluent Conjecture B for large $t$, one faces a conceptual problem of "good" choice of the monodromy parameter defining the accessory parameter function, as well as more technical issue of its efficient computation. We propose to replace the Floquet exponent $\sigma$ by the all-order Bohr-Sommerfeld (BS) period

$$
v=\frac{1}{2 \pi i} \oint_{\gamma} S_{\text {odd }}(z) d z, \quad S_{\text {odd }}(z)=\sum_{n=0}^{\infty} S_{2 n-1}(z),
$$

for a suitably chosen closed contour $\gamma$, with $S_{-1}(z)=\sqrt{V(z)}$ and $S_{n}^{\prime}+\sum_{k=-1}^{n+1} S_{k} S_{n-k}=0$. The computation of BS periods is a standard tool of $\mathscr{N}=2$ SUSY gauge theory $[\mathrm{MM}, \mathrm{HM}]$; see also [BD, GGM] and references therein. In the gauge theory context, the role of the Planck constant is played by one of the Nekrasov deformation parameters $\epsilon_{1,2}$ (the other one being 0 in the NS limit). In our setup, however, the relevant WKB parameter is the inverse coupling $\frac{1}{t}$.

We focus on two examples: (a) confluent and (b) biconfluent Heun equations $\mathrm{H}_{\mathrm{V}}$ and $\mathrm{H}_{\mathrm{IV}}$. Inverting the relation $v=v(\mathscr{E}, t)$, it is fairly easy to obtain accessory parameter $\mathscr{E}=\mathscr{E}^{[\mathrm{BS}]}(t \mid v)$ in the form of a formal asymptotic series in $\frac{1}{t}$. These series are then identified with classical confluent CBs via Conjectures 3.1 and 3.2. We find that, similarly to 1.9 and 1.12 ,

$$
\mathscr{E}_{\mathrm{V}}^{[\mathrm{BS}]}(t \mid v)=t \frac{\partial}{\partial t} \mathscr{U}_{N_{f}=3}(t), \quad \mathscr{E}_{\mathrm{IV}}^{[\mathrm{BS}]}(t \mid v)=\frac{\partial}{\partial t} \tilde{\mathcal{U}}(t),
$$

where $\mathscr{U}_{N_{f}=3}(t)$ and $\tilde{\mathscr{U}}(t)$ are classical counterparts of CBs introduced in [GT, Na1. This observation is consistent with a conjectural relation between conformal blocks of the 2nd kind and partition functions of strongly coupled gauge/Argyres-Douglas theories [GT, BLMST, NU], and may be viewed as a quasiclassical variant of the AGT or Bethe/gauge correspondence.

\section{Classical confluent conformal blocks}

\subsection{Regular conformal blocks and their classical limit}

Regular CBs are matrix elements of compositions of vertex operators between states in the highest weight representations of the Virasoro algebra

$$
\left[L_{m}, L_{n}\right]=(m-n) L_{m+n}+\frac{c}{12} n\left(n^{2}-1\right) \delta_{m+n, 0} .
$$

A highest weight representation $\mathcal{V}_{\Delta}$ and its dual $\mathcal{V}_{\Delta}^{*}$ are generated from the states $|\Delta\rangle,\langle\Delta|$ defined by

$$
\begin{array}{ll}
L_{0}|\Delta\rangle=\Delta|\Delta\rangle, & L_{n>0} \mid \Delta=0, \\
\langle\Delta| L_{0}=\Delta\langle\Delta|, & \langle\Delta| L_{n<0}=0 .
\end{array}
$$


There is a canonical bilinear pairing $\langle\mid\rangle: \mathcal{V}_{\Delta}^{*} \times \mathbb{V}_{\Delta} \rightarrow \mathbb{C}$ satisfying $\left\langle u\left|L_{n} \cdot\right| v\right\rangle=\left\langle u\left|\cdot L_{n}\right| v\right\rangle$ for all $\left\langle u\left|\in \mathscr{V}_{\Delta}^{*},\right| v\right\rangle \in \mathbb{V}_{\Delta}$ and $n \in \mathbb{Z}$. It will be normalized by $\langle\Delta \mid \Delta\rangle=1$. The primary regular vertex operators $V_{\Delta_{3}, \Delta_{1}}^{\Delta_{2}}(t): \mathscr{V}_{\Delta_{1}} \rightarrow \mathscr{V}_{\Delta_{3}}$ are defined by the commutation relation

$$
\left[L_{n}, V_{\Delta_{3}, \Delta_{1}}^{\Delta_{2}}(t)\right]=t^{n}\left(t \frac{\partial}{\partial t}+(n+1) \Delta_{2}\right) V_{\Delta_{3}, \Delta_{1}}^{\Delta_{2}}(t) .
$$

It determines $V_{\Delta_{3}, \Delta_{1}}^{\Delta_{2}}(t)$ uniquely up to normalization (multiplicative constant independent of $t$ ), which we further fix by setting $\left\langle\Delta_{3}\left|V_{\Delta_{3}, \Delta_{1}}^{\Delta_{2}}(t)\right| \Delta_{1}\right\rangle=t^{\Delta_{3}-\Delta_{2}-\Delta_{1}}$. Spherical 4-point conformal block 1.5] is defined as

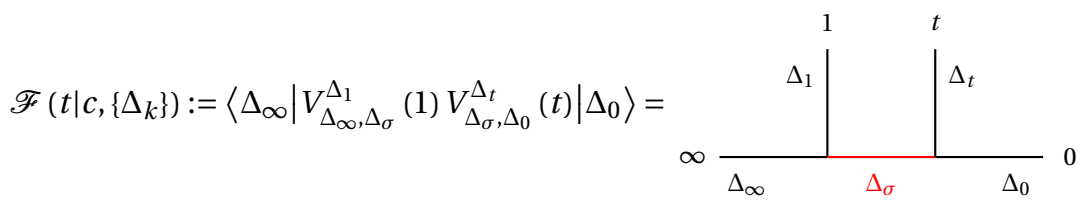

The low-order expansion coefficients in $\mathscr{F}\left(t \mid c,\left\{\Delta_{k}\right\}\right)=t^{\Delta_{\sigma}-\Delta_{0}-\Delta_{t}}\left[1+\sum_{n=1}^{\infty} \mathscr{F}_{n}\left(c,\left\{\Delta_{k}\right\}\right) t^{n}\right]$ can be computed

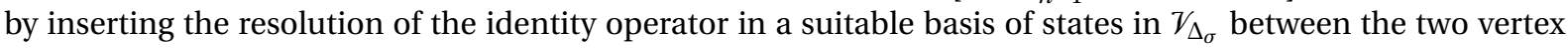
operators in 2.4. For example, one has

$$
\begin{aligned}
\mathscr{F}_{1}\left(c,\left\{\Delta_{k}\right\}\right) & =\frac{\left(\Delta_{\sigma}-\Delta_{0}+\Delta_{t}\right)\left(\Delta_{\sigma}-\Delta_{\infty}+\Delta_{1}\right)}{2 \Delta_{\sigma}}, \\
\mathscr{F}_{2}\left(c,\left\{\Delta_{k}\right\}\right) & =\frac{\left(\Delta_{\sigma}-\Delta_{0}+\Delta_{t}\right)\left(\Delta_{\sigma}-\Delta_{0}+\Delta_{t}+1\right)\left(\Delta_{\sigma}-\Delta_{\infty}+\Delta_{1}\right)\left(\Delta_{\sigma}-\Delta_{\infty}+\Delta_{1}+1\right)}{4 \Delta_{\sigma}\left(1+2 \Delta_{\sigma}\right)}+ \\
& +\frac{\left(1+2 \Delta_{\sigma}\right)\left(\Delta_{0}+\Delta_{t}+\frac{\Delta_{\sigma}\left(\Delta_{\sigma}-1\right)-3\left(\Delta_{0}-\Delta_{t}\right)^{2}}{1+2 \Delta_{\sigma}}\right)\left(\Delta_{\infty}+\Delta_{1}+\frac{\Delta_{\sigma}\left(\Delta_{\sigma}-1\right)-3\left(\Delta_{\infty}-\Delta_{1}\right)^{2}}{1+2 \Delta_{\sigma}}\right)}{2\left(1-4 \Delta_{\sigma}\right)^{2}+2(c-1)\left(1+2 \Delta_{\sigma}\right)} .
\end{aligned}
$$

The form of $\mathscr{F}_{n}$ 's becomes quite involved with the growth of $n$ but all coefficients can still be explicitly computed for arbitrary $n$ thanks to the AGT relation. For concrete formulas, see e.g. AFLT (or [LNR Eqs. (1.2)(1.4)], whose notation is close to the present paper).

The quasiclassical limit described by $1.6-1.8$ more precisely means that one should consider $\frac{6}{c} \ln \mathscr{F}\left(t \mid c,\left\{\Delta_{k}\right\}\right)$ as a formal series in $t$, and every coefficient of this series has a finite limit (Conjecture A). E.g. from 2.5a $-2.5 \mathrm{~b}$ we find

$$
\begin{aligned}
\mathscr{W}_{1}\left(\left\{\delta_{k}\right\}\right) & =\frac{\left(\delta_{\sigma}-\delta_{0}+\delta_{t}\right)\left(\delta_{\sigma}-\delta_{\infty}+\delta_{1}\right)}{2 \delta_{\sigma}} \\
\mathscr{W}_{2}\left(\left\{\delta_{k}\right\}\right) & =\frac{\left(\delta_{\sigma}-\delta_{0}+\delta_{t}\right)^{2}\left(\delta_{\sigma}-\delta_{\infty}+\delta_{1}\right)^{2}}{8 \delta_{\sigma}^{2}}\left[\frac{1}{\delta_{\sigma}-\delta_{0}+\delta_{t}}+\frac{1}{\delta_{\sigma}-\delta_{\infty}+\delta_{1}}-\frac{1}{2 \delta_{\sigma}}\right]+ \\
& +\frac{\left(\delta_{\sigma}^{2}+2 \delta_{\sigma}\left(\delta_{0}+\delta_{t}\right)-3\left(\delta_{0}-\delta_{t}\right)^{2}\right)\left(\delta_{\sigma}^{2}+2 \delta_{\sigma}\left(\delta_{\infty}+\delta_{1}\right)-3\left(\delta_{\infty}-\delta_{1}\right)^{2}\right)}{16 \delta_{\sigma}^{2}\left(4 \delta_{\sigma}+3\right)}
\end{aligned}
$$

Already at the order $O\left(t^{2}\right)$, the existence of the limit is not immediately obvious and involves a cancellation of separately divergent contributions from $\mathscr{F}_{1}$ and $\mathscr{F}_{2}$.

In the discussion of confluent limits, it will be occasionally convenient to use the Liouville CFT parameterization of the Virasoro central charge and conformal dimensions,

$$
c=1+6\left(b+b^{-1}\right)^{2}, \quad \Delta=\frac{c-1}{24}+P^{2} .
$$

The classical limit of the regular CB corresponds to setting $P_{k}=i b^{-1} \theta_{k}(k=0,1, t, \infty, \sigma)$ and sending $b$ to 0 , so that $c \sim 6 b^{-2}$ and $\Delta_{k} \sim b^{-2} \delta_{k}$ with $\delta_{k}=\frac{1}{4}-\theta_{k}^{2}$. When it will be useful to indicate the dependence of CBs such as $\mathscr{F}\left(t \mid c,\left\{\Delta_{k}\right\}\right)$ and $\mathscr{W}\left(t \mid\left\{\delta_{k}\right\}\right)$ on various conformal dimensions more explicitly, we will accordingly use a notation such as $\mathscr{F}\left(\begin{array}{lll}P_{1} & P_{t} \\ P_{\infty} & P_{\sigma} & P_{0}\end{array} ; t\right)$ and $\mathbb{W}\left(\begin{array}{ccc}\theta_{1} & \theta_{t} \\ \theta_{\infty} & \theta_{0}\end{array} ; t\right)$. 


\subsection{Confluent conformal blocks of the 1st kind}

\subsubsection{Whittaker modules}

In the confluent case, in addition to the highest weight representations for the Virasoro algebra, one also needs to consider Whittaker modules. A Whittaker module $\mathcal{V}_{\vec{\lambda}}^{W}$ of rank $r$ is generated from a joint eigenstate $|\vec{\lambda}\rangle=$ $\left|\lambda_{r}, \ldots, \lambda_{2 r}\right\rangle$ of Virasoro generators $L_{r}, \ldots, L_{2 r}$ Gai BMT GT:

$$
L_{n}\left|\lambda_{r}, \ldots, \lambda_{2 r}\right\rangle=\left\{\begin{array}{cl}
\lambda_{n}\left|\lambda_{r}, \ldots, \lambda_{2 r}\right\rangle, & r \leq n \leq 2 r, \\
0, & n>2 r,
\end{array}\right.
$$

which is called a Whittaker vector. Note that $e^{s L_{n}}|\vec{\lambda}\rangle$ with $n=0, \ldots, r-1$ and $s \in \mathbb{C}$ is again a Whittaker vector. For example,

$$
t^{L_{0}}\left|\lambda_{r}, \ldots, \lambda_{2 r}\right\rangle \sim\left|t^{r} \lambda_{r}, \ldots, t^{2 r} \lambda_{2 r}\right\rangle,
$$

This property of $e^{s L_{n}}|\vec{\lambda}\rangle$ will be used later to fix some of the eigenvalues in the definitions of confluent CBs. The present subsection is concerned only with Whittaker modules of rank 1 . In this case, there is a canonical bilinear pairing $\langle\mid\rangle: \mathscr{V}_{\left(\lambda_{1}, \lambda_{2}\right)}^{W, *} \times \mathscr{V}_{\Delta} \rightarrow \mathbb{C}$ which is uniquely determined by the condition $\left\langle w\left|\cdot L_{n}\right| v\right\rangle=\left\langle w\left|L_{n} \cdot\right| \nu\right\rangle$ for all $\left\langle w\left|\in \mathcal{V}_{\left(\lambda_{1}, \lambda_{2}\right)}^{W, *},\right| v\right\rangle \in \mathcal{V}_{\Delta}$ and $n \in \mathbb{Z}$, and normalization $\left\langle\lambda_{1}, \lambda_{2} \mid \Delta\right\rangle=1$.

2.2.2 $N_{f}=3$

The confluent CB of the 1st kind, AGT-dual to the $N_{f}=3 \mathrm{SU}$ (2) Nekrasov partition function, can be defined as the matrix element

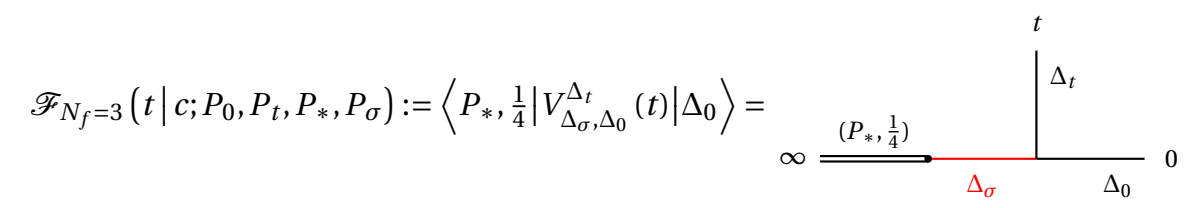

Its small $t$ expansion may be found by expanding $V_{\Delta_{\sigma}, \Delta_{0}}^{\Delta_{t}}(t)\left|\Delta_{0}\right\rangle$ in a suitable basis of $\mathcal{V}_{\Delta_{\sigma}}$ and computing the pairing with $\left\langle P_{*}, \frac{1}{4}\right|$. The first few coefficients are explicitly given by

$$
\begin{aligned}
& \mathscr{F}_{N_{f}=3}\left(t \mid c ;\left\{P_{k}\right\}\right)=t^{\Delta_{\sigma}-\Delta_{0}-\Delta_{t}}\left[1+\frac{\left(\Delta_{\sigma}-\Delta_{0}+\Delta_{t}\right) P_{*}}{2 \Delta_{\sigma}} t+\right. \\
& \left.+\left(\frac{\left(\Delta_{\sigma}-\Delta_{0}+\Delta_{t}\right)\left(\Delta_{\sigma}-\Delta_{0}+\Delta_{t}+1\right) P_{*}^{2}}{\Delta_{\sigma}\left(1+2 \Delta_{\sigma}\right)}+\frac{\left(\Delta_{0}+\Delta_{t}+\frac{\Delta_{\sigma}\left(\Delta_{\sigma}-1\right)-3\left(\Delta_{0}-\Delta_{t}\right)^{2}}{1+2 \Delta_{\sigma}}\right)\left(1+2 \Delta_{\sigma}-6 P_{*}^{2}\right)}{16 \Delta_{\sigma}^{2}-10 \Delta_{\sigma}+c\left(1+2 \Delta_{\sigma}\right)}\right) \frac{t^{2}}{4}+O\left(t^{3}\right)\right] .
\end{aligned}
$$

This series can also be obtained by taking termwise confluent limit of the expansion 1.5 where 2 of the 5 conformal dimensions become infinite in a correlated way:

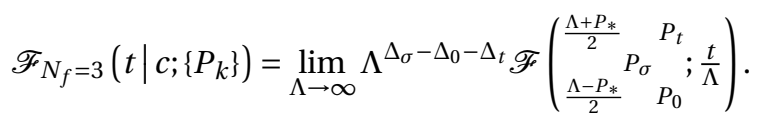

The exponentiation conjecture for the quasiclassical asymptotics of the confluent $\mathrm{CB}[2.10$ is similar to the regular case but involves in addition a rescaling of $t$. More specifically,

$$
\mathscr{F}_{N_{f}=3}\left(\frac{i t}{b} \mid c ; \frac{i \theta_{0}}{b}, \frac{i \theta_{t}}{b}, \frac{i \theta_{*}}{b}, \frac{i \sigma}{b}\right) \stackrel{b \rightarrow 0}{\sim} \text { const } \cdot \exp \left\{b^{-2} \mathscr{W}_{N_{f}=3}\left(t \mid \theta_{0}, \theta_{t}, \theta_{*}, \sigma\right)\right\},
$$

where the constant (independent of $t$ ) prefactor does not necessarily have a finite limit as $b \rightarrow 0$. Using [2.11], one finds

$$
\begin{aligned}
& \mathscr{W}_{N_{f}=3}\left(t \mid \theta_{0}, \theta_{t}, \theta_{*}, \sigma\right)=\left(\delta_{\sigma}-\delta_{0}-\delta_{t}\right) \ln t-\frac{\left(\delta_{\sigma}-\delta_{0}+\delta_{t}\right) \theta_{*}}{2 \delta_{\sigma}} t+ \\
& +\left[\frac{\left(\delta_{\sigma}^{2}-\left(\delta_{0}-\delta_{t}\right)^{2}\right) \theta_{*}^{2}}{16 \delta_{\sigma}^{3}}-\frac{\left(3 \theta_{*}^{2}+\delta_{\sigma}\right)\left(\delta_{\sigma}^{2}+2 \delta_{\sigma}\left(\delta_{0}+\delta_{t}\right)-3\left(\delta_{0}-\delta_{t}\right)^{2}\right)}{16 \delta_{\sigma}^{2}\left(3+4 \delta_{\sigma}\right)}\right] t^{2}+O\left(t^{3}\right),
\end{aligned}
$$

where $\delta_{0}=\frac{1}{4}-\theta_{0}^{2}, \delta_{t}=\frac{1}{4}-\theta_{0}^{2}, \delta_{\sigma}=\frac{1}{4}-\sigma^{2}$ as above. 
2.2.3 $N_{f}=0,1,2$

Let $\Pi_{\Delta}$ denote the identity operator on $\mathcal{V}_{\Delta}$, considered as an element of $\mathcal{V}_{\Delta} \otimes \mathcal{V}_{\Delta}^{*}$. For instance, writing the contributions of descendant states up to level 2, we have

$$
\Pi_{\Delta}=|\Delta\rangle\left\langle\Delta \left|+\frac{L_{-1}|\Delta\rangle\langle\Delta| L_{1}}{2 \Delta}+\left(L_{-1}^{2}|\Delta\rangle L_{-2}|\Delta\rangle\right)\left(\begin{array}{cc}
4 \Delta(2 \Delta+1) & 6 \Delta \\
6 \Delta & 4 \Delta+\frac{c}{2}
\end{array}\right)^{-1}\left(\begin{array}{l}
\langle\Delta| L_{1}^{2} \\
\langle\Delta| L_{2}
\end{array}\right)+\ldots\right.\right.
$$

Confluent CBs of the 1st kind corresponding to $N_{f}=0,1,2$ are defined as matrix elements

$$
\begin{aligned}
\mathscr{F}_{N_{f}=2}\left(t \mid c ; P_{\star}, P_{*}, P_{\sigma}\right) & :=\left\langle P_{*}, \frac{1}{4}\left|t^{L_{0}} \Pi_{\Delta_{\sigma}}\right| P_{\star}, \frac{1}{4}\right\rangle, \\
\mathscr{F}_{N_{f}=1}\left(t \mid c ; P_{*}, P_{\sigma}\right) & :=\left\langle P_{*}, \frac{1}{4}\left|t^{L_{0}} \Pi_{\Delta_{\sigma}}\right| 1,0\right\rangle, \\
\mathscr{F}_{N_{f}=0}\left(t \mid c ; P_{\sigma}\right) & :=\left\langle 1,0\left|t^{L_{0}} \Pi_{\Delta_{\sigma}}\right| 1,0\right\rangle .
\end{aligned}
$$

Small $t$ expansions of these CBs can be computed using 2.15:

$$
\begin{aligned}
\mathscr{F}_{N_{f}=2}\left(t \mid c ; P_{\star}, P_{*}, P_{\sigma}\right) & =t^{\Delta_{\sigma}}\left[1+\frac{P_{*} P_{\star}}{2 \Delta_{\sigma}} t+\frac{P_{\star}^{2} P_{*}^{2}\left(\frac{c}{4 \Delta_{\sigma}}+2\right)-\frac{3}{4}\left(P_{\star}^{2}+P_{*}^{2}\right)+\frac{1}{8}\left(1+2 \Delta_{\sigma}\right)}{16 \Delta_{\sigma}^{2}-10 \Delta_{\sigma}+c\left(1+2 \Delta_{\sigma}\right)} t^{2}+O\left(t^{3}\right)\right], \\
\mathscr{F}_{N_{f}=1}\left(t \mid c ; P_{*}, P_{\sigma}\right) & =t^{\Delta_{\sigma}}\left[1+\frac{P_{*}}{2 \Delta_{\sigma}} t+\frac{P_{*}^{2}\left(\frac{c}{4 \Delta_{\sigma}}+2\right)-\frac{3}{4}}{16 \Delta_{\sigma}^{2}-10 \Delta_{\sigma}+c\left(1+2 \Delta_{\sigma}\right)} t^{2}+O\left(t^{3}\right)\right], \\
\mathscr{F}_{N_{f}=0}\left(t \mid c ; P_{\sigma}\right) & =t^{\Delta_{\sigma}}\left[1+\frac{t}{2 \Delta_{\sigma}}+\frac{\frac{c}{4 \Delta_{\sigma}}+2}{16 \Delta_{\sigma}^{2}-10 \Delta_{\sigma}+c\left(1+2 \Delta_{\sigma}\right)} t^{2}+O\left(t^{3}\right)\right] .
\end{aligned}
$$

The same expansions are also generated by a chain of termwise confluent limits:

$$
\begin{aligned}
\mathscr{F}_{N_{f}=2}\left(t \mid c ; P_{\star}, P_{*}, P_{\sigma}\right) & =\lim _{\Lambda \rightarrow \infty} \Lambda^{\Delta_{\sigma}-\Delta_{0}-\Delta_{t}} t^{\Delta_{0}+\Delta_{t}} \mathscr{F}_{N_{f}=3}\left(\frac{t}{\Lambda} \mid c ; P_{0}=\frac{\Lambda-P_{\star}}{2}, P_{t}=\frac{\Lambda+P_{\star}}{2}, P_{*}, P_{\sigma}\right), \\
\mathscr{F}_{N_{f}=1}\left(t \mid c ; P_{*}, P_{\sigma}\right) & =\lim _{P_{\star} \rightarrow \infty} P_{\star}^{\Delta_{\sigma}} \mathscr{F} N_{f}=2\left(\frac{t}{P_{\star}} \mid c ; P_{\star}, P_{*}, P_{\sigma}\right), \\
\mathscr{F}_{N}=0 & \left(t \mid c ; P_{\sigma}\right)=\lim _{P_{*} \rightarrow \infty} P_{*}^{\Delta_{\sigma}} \mathscr{F}_{N_{f}=1}\left(\frac{t}{P_{*}} \mid c ; P_{*}, P_{\sigma}\right) .
\end{aligned}
$$

The exponentiation of the confluent CBs 2.16] is described by conjectural formulas analogous to [2.13]:

$$
\begin{gathered}
\mathscr{F}_{N_{f}=2}\left(-\frac{t}{b^{2}} \mid c ; \frac{i \theta_{\star}}{b}, \frac{i \theta_{*}}{b}, \frac{i \sigma}{b}\right) \stackrel{b \rightarrow 0}{\sim} \text { const } \cdot \exp \left\{b^{-2} \mathscr{W}_{N_{f}=2}\left(t \mid \theta_{\star}, \theta_{*}, \sigma\right)\right\}, \\
\mathscr{F}_{N_{f}=1}\left(-\frac{i t}{b^{3}} \mid c ; \frac{i \theta_{*}}{b}, \frac{i \sigma}{b}\right) \stackrel{b \rightarrow 0}{\sim} \text { const } \cdot \exp \left\{b^{-2} \mathscr{W}_{N_{f}=1}\left(t \mid \theta_{*}, \sigma\right)\right\}, \\
\mathscr{F}_{N_{f}=0}\left(\frac{t}{b^{4}} \mid c ; \frac{i \sigma}{b}\right) \stackrel{b \rightarrow 0}{\sim} \text { const } \cdot \exp \left\{b^{-2} \mathscr{W}_{N_{f}=0}(t \mid \sigma)\right\}
\end{gathered}
$$

These confluent variants of Conjecture A are of course straightforward to check at low orders in $t$. We record below only a few terms in the expansions of $\mathbb{W}_{N_{f}=0,1,2}(t)$ which easily follow from 2.17,

$$
\begin{aligned}
\mathbb{W}_{N_{f}=2}\left(t \mid \theta_{\star}, \theta_{*}, \sigma\right) & =\delta_{\sigma} \ln t+\frac{\theta_{\star} \theta_{*}}{2 \delta_{\sigma}} t+\left(\frac{\left(3 \theta_{*}^{2}+\delta_{\sigma}\right)\left(3 \theta_{\star}^{2}+\delta_{\sigma}\right)}{16 \delta_{\sigma}^{2}\left(3+4 \delta_{\sigma}\right)}-\frac{\theta_{*}^{2} \theta_{\star}^{2}}{16 \delta_{\sigma}^{3}}\right) t^{2}+O\left(t^{3}\right), \\
W_{N_{f}=1}\left(t \mid \theta_{*}, \sigma\right) & =\delta_{\sigma} \ln t+\frac{\theta_{*}}{2 \delta_{\sigma}} t+\frac{\left(5 \delta_{\sigma}-3\right) \theta_{*}^{2}+3 \delta_{\sigma}^{2}}{16 \delta_{\sigma}^{3}\left(3+4 \delta_{\sigma}\right)} t^{2}+O\left(t^{3}\right), \\
\mathscr{W}_{N_{f}=0}(t \mid \sigma) & =\delta_{\sigma} \ln t+\frac{t}{2 \delta_{\sigma}}+\frac{5 \delta_{\sigma}-3}{16 \delta_{\sigma}^{3}\left(3+4 \delta_{\sigma}\right)} t^{2}+O\left(t^{3}\right) .
\end{aligned}
$$

Note that the expansions of $\mathbb{W}_{N_{f}<4}(t)$ can also be obtained directly from the regular quasiclassical CB $\mathbb{W}\left(t \mid\left\{\delta_{k}\right\}\right)$ by the same sequence of termwise limits as in (1.11); in other words, for CBs of the 1st kind the quasiclassical limit commutes with confluence. 


\subsection{Confluent conformal blocks of the 2nd kind}

\subsubsection{Irregular vertex operators}

Let us recall the construction of a (dual) irregular vertex operator $V_{\vec{\lambda}, \vec{\lambda}^{\prime}}^{\Delta}(t): \mathcal{V}_{\vec{\lambda}}^{W, *} \rightarrow \mathcal{V}_{\vec{\lambda}^{\prime}}^{W, *}$ from [Na1]. It is uniquely determined by the following properties:

- Whittaker modules $\mathcal{V}_{\vec{\lambda}}^{W, *}, \mathcal{V}_{\vec{\lambda}^{\prime}}^{W, *}$ have the same rank $r$ and satisfy a genericity condition $\lambda_{2 r}, \lambda_{2 r}^{\prime} \neq 0$.

- The operator $V_{\vec{\lambda}, \vec{\lambda}^{\prime}}^{\Delta}(t)$ has the same commutation relations 2.3 with the Virasoro generators as the usual vertex operator:

$$
\left[L_{n}, V_{\vec{\lambda}, \vec{\lambda}^{\prime}}^{\Delta}(t)\right]=t^{n}\left(t \frac{\partial}{\partial t}+(n+1) \Delta\right) V_{\vec{\lambda}, \vec{\lambda}^{\prime}}^{\Delta}(t) .
$$

This is essentially an expression of the local conformal invariance.

- Most importantly, there is a "normalization" condition

$$
\left\langle\lambda_{r}, \ldots, \lambda_{2 r}\right| V_{\vec{\lambda}, \vec{\lambda}^{\prime}}^{\Delta}(t)=t^{\alpha} \exp \left\{\sum_{k=1}^{r} \beta_{k} t^{k}\right\}\left[\left\langle\lambda_{r}^{\prime}, \ldots, \lambda_{2 r}^{\prime}\right|+\sum_{n=1}^{\infty} t^{-n}\left\langle w_{n}^{\prime}\right|\right],
$$

where the expression in the square brackets is understood as a formal series in $\mathcal{V}_{\vec{\lambda}^{\prime}}^{W, *} \otimes \mathbb{C}\left[\left[t^{-1}\right]\right]$.

The parameters $\vec{\lambda}^{\prime}, \alpha, \beta_{1}, \ldots, \beta_{r-1}$ on the right of 2.22 as well as all coefficients $\left\langle w_{n}^{\prime}\right| \in \mathcal{V}_{\vec{\lambda}^{\prime}}^{W, *}$ of the formal series are determined by $\vec{\lambda}, \Delta$ and $\beta_{r}$. In particular, one has

$$
\lambda_{k}^{\prime}= \begin{cases}\lambda_{k}, & k=r+1, \ldots, 2 r, \\ \lambda_{r}-r \beta_{r}, & k=r .\end{cases}
$$

\subsubsection{Confluent conformal blocks of type $\mathscr{D}$}

The canonical pairing between the Virasoro highest weight modules and Whittaker modules of rank 1, already used in the previous subsection, allows to define [Nal] a class of 3-point confluent CBs of the 2nd kind:

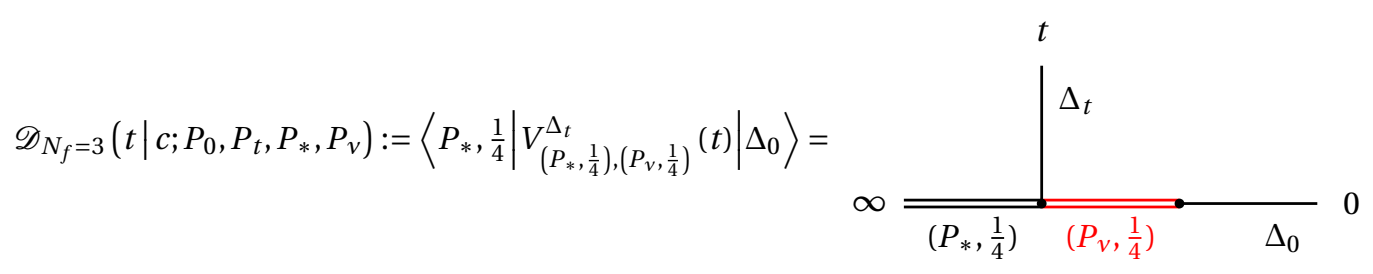

They will be referred to as confluent CBs of type $\mathscr{D}$. The coefficients of the large $t$ expansion of these CBs,

$$
\mathscr{D}_{N_{f}=3}\left(t \mid c ;\left\{P_{k}\right\}\right)=t^{2 P_{v}\left(P_{*}-P_{v}\right)} e^{\left(P_{*}-P_{v}\right) t}\left[1+\sum_{n=1}^{\infty} \mathscr{D}_{n}\left(c ;\left\{P_{k}\right\}\right) t^{-n}\right],
$$

as well as the exponent $\alpha=2 P_{v}\left(P_{*}-P_{v}\right)$, are thus fixed by Virasoro symmetry. Two lowest order coefficients can be deduced from the expressions for $\left\langle w_{1}^{\prime}\right|,\left\langle w_{2}^{\prime}\right|$ in [Na1 Appendix A.2.1]. One has

$$
\begin{aligned}
\mathscr{D}_{1}\left(c ;\left\{P_{k}\right\}\right) & =-4 P_{v}^{3}+6 P_{v}^{2} P_{*}+2 P_{v}\left(\Delta_{0}+\Delta_{t}-P_{*}^{2}\right)-2 \Delta_{0} P_{*}, \\
\mathscr{D}_{2}\left(c ;\left\{P_{k}\right\}\right) & =\frac{1}{2} \mathscr{D}_{1}\left(c ;\left\{P_{k}\right\}\right)^{2}+2\left(\Delta_{0}+\left(P_{*}-3 P_{v}\right) P_{v}\right)\left(\Delta_{t}-\left(2 P_{*}-3 P_{v}\right)\left(P_{*}-P_{v}\right)\right)+ \\
& +\left(2\left(P_{*}-2 P_{v}\right)^{2}+\frac{c-1}{3}\right) P_{v}\left(P_{*}-P_{v}\right) .
\end{aligned}
$$

The algebraic computation of the coefficients $\left\{\mathscr{D}_{k}\right\}$ becomes quite lengthy at higher orders. However, all of them remain explicitly computable (at least in principle) since $\mathscr{D}_{N_{f}=3}\left(t \mid c ;\left\{P_{k}\right\}\right)$ can be identified [LNR] with a particular limit of the regular $u$-channel CB suggested in [GT]. Namely,

$$
1+\sum_{n=1}^{\infty} \mathscr{D}_{n}\left(c ;\left\{P_{k}\right\}\right) t^{-n}=\lim _{\Lambda \rightarrow \infty}\left(\frac{\Lambda}{t}\right)^{\Delta_{t}-\left(P_{*}-P_{v}\right)\left(\Lambda-P_{v}\right)}\left(1-\frac{\Lambda}{t}\right)^{\left(P_{*}-P_{v}\right)\left(\Lambda+P_{v}\right)+\Delta_{t}} \mathscr{F}\left(\begin{array}{c}
\frac{\Lambda+P_{*}}{2} \\
\frac{\Lambda+P_{*}}{2}-P_{v} \\
P_{0}
\end{array} ; \frac{\Lambda-P_{*}}{2}\right),
$$


where the last two factors under the limit are interpreted as formal series in $\frac{1}{t}$ (note that only the product of these series admits a finite limit as $\Lambda \rightarrow \infty$ while the coefficients of each of them diverge when treated separately). Another difference from the confluent limit leading to CBs of the 1st kind is that the intermediate dimension also goes to $\infty$. The relation 2.27) of course reproduces 2.26a, 2.26b) and allows to go to any desired order. For instance,

$$
\begin{aligned}
& \mathscr{D}_{3}\left(c ;\left\{P_{k}\right\}\right)=\mathscr{D}_{1}\left(c ;\left\{P_{k}\right\}\right) \mathscr{D}_{2}\left(c ;\left\{P_{k}\right\}\right)-\frac{1}{3} \mathscr{D}_{1}\left(c ;\left\{P_{k}\right\}\right)^{3}-\left(11 P_{v}\left(P_{*}-P_{v}\right)-\frac{5}{3} P_{*}^{2}+\frac{13}{6}\left(\Delta_{0}+\Delta_{t}\right)\right) \mathscr{D}_{1}\left(c ;\left\{P_{k}\right\}\right)+ \\
& +\frac{11}{3}\left(\Delta_{t}-\Delta_{0}-2 P_{*}\left(P_{*}-2 P_{v}\right)\right)\left(\left(P_{*}-P_{v}\right) \Delta_{0}+P_{v} \Delta_{t}+P_{*} P_{v}\left(P_{*}-P_{v}\right)\right)+\frac{2}{3}(c-2)\left(\left(P_{*}-P_{v}\right) \Delta_{0}-P_{v} \Delta_{t}\right)+ \\
& +\frac{1}{9}\left(66 P_{*}^{2}+17 c-23\right)\left(P_{*}-2 P_{v}\right)\left(P_{*}-P_{v}\right) P_{v} .
\end{aligned}
$$

The above expressions for $\mathscr{D}_{2,3}$ are organized in a way which makes manifest the exponentiation of CBs of type $\mathscr{D}$ at low orders. We thus arrive at the following confluent variant of Conjecture A, cf 2.13).

Conjecture 2.1. Confluent conformal blocks of type D exponentiate as

$$
\mathscr{D}_{N_{f}=3}\left(\frac{i t}{b} \mid c ; \frac{i \theta_{0}}{b}, \frac{i \theta_{t}}{b}, \frac{i \theta_{*}}{b}, \frac{i v}{b}\right) \stackrel{b \rightarrow 0}{\sim} \text { const } \cdot \exp \left\{b^{-2} \mathscr{U}_{N_{f}=3}\left(t \mid \theta_{0}, \theta_{t}, \theta_{*}, v\right)\right\},
$$

with

$$
\mathscr{U}_{N_{f}=3}\left(t \mid \theta_{0}, \theta_{t}, \theta_{*}, v\right)=\left(v-\theta_{*}\right) t+2 v\left(v-\theta_{*}\right) \ln t+\sum_{n=1}^{\infty} \mathscr{U}_{n}\left(\theta_{0}, \theta_{t}, \theta_{*}, v\right) t^{-n} .
$$

We have checked this claim up to order $O\left(t^{-4}\right)$. The coefficients of the classical CB of type $\mathscr{D}$ are readily obtained from those of $\mathscr{D}_{N_{f}=3}(t)$. E.g. from [2.26) it follows that

$$
\begin{aligned}
& \mathscr{U}_{1}\left(\theta_{0}, \theta_{t}, \theta_{*}, v\right)=4 v^{3}-6 v^{2} \theta_{*}+2 v\left(\delta_{0}+\delta_{t}+\theta_{*}^{2}\right)-2 \delta_{0} \theta_{*}, \\
& \mathscr{U}_{2}\left(\theta_{0}, \theta_{t}, \theta_{*}, v\right)=-2\left(\delta_{0}-v\left(\theta_{*}-3 v\right)\right)\left(\delta_{t}+\left(2 \theta_{*}-3 v\right)\left(\theta_{*}-v\right)\right)-2 v\left(\theta_{*}-v\right)\left(\left(\theta_{*}-2 v\right)^{2}-1\right) .
\end{aligned}
$$

Assuming that the quasiclassical limit commutes with confluence for the CBs of the 2nd kind as well, we can alternatively compute these coefficients from a quasiclassical version of 2.27,

$$
\mathscr{U}_{n}\left(\theta_{0}, \theta_{t}, \theta_{*}, v\right)=\lim _{\Lambda \rightarrow \infty} \Lambda^{n}\left[\mathscr{W}_{n}\left(\begin{array}{ccc}
\frac{\Lambda+\theta_{*}}{2} & \theta_{t} \\
& \frac{\Lambda+\theta_{*}}{2}-v \\
\theta_{0} & \frac{\Lambda-\theta_{*}}{2}
\end{array}\right)-\frac{\delta_{t}-\left(\theta_{*}-v\right)(\Lambda+v)}{n}\right],
$$

where $\mathbb{W}_{n}$ 's denote the expansion coefficients of the regular classical CB.

\subsubsection{Confluent conformal blocks of type $\mathscr{G}$}

There is no obvious canonical pairing between Whittaker modules of rank $r \geq 2$ and highest weight representations. For example, a pairing such as $\left\langle\vec{\lambda}\left|\cdot L_{-1}^{k}\right| \Delta\right\rangle$ of the Whittaker vector and a descendant state in $\bigvee_{\Delta}$ for $r>1$ cannot be reduced to $\langle\vec{\lambda} \mid \Delta\rangle$ since $\langle\vec{\lambda}|$ is not an eigenstate of $L_{-1}$. A notable exception concerns the pairing of Whittaker modules of rank 2 with an irreducible highest weight module $V_{0}$ (as in this case $L_{-1}|0\rangle=0$ ). This allows to define [Na1] confluent CBs

$$
\mathscr{G}\left(t \mid c ; P_{0}, P_{\bullet}, P_{v}\right)=\left\langle P_{\bullet}, 0, \frac{1}{4}\left|V_{\left(P_{\bullet}, 0, \frac{1}{4}\right),\left(P_{\bullet}-P_{v}, 0, \frac{1}{4}\right)}^{\left.\Delta_{0}\right)}(t)\right| 0\right\rangle=\underbrace{\Delta_{0}}_{\underset{\left(P_{\bullet}, 0, \frac{1}{4}\right)}{\Delta_{\left(P_{\bullet}-P_{v}, 0, \frac{1}{4}\right)}}} 0
$$

They will be referred to as CBs of the 2 nd kind of type $\mathscr{G}$. Setting the eigenvalues $\lambda_{3}, \lambda_{4}$ to 0 and $\frac{1}{4}$ involves no loss of generality thanks to translation and dilatation symmetry of (2.33).

The large $t$ expansion of the conformal block $\mathscr{G}\left(t \mid c ;\left\{P_{k}\right\}\right)$ has the form

$$
\mathscr{G}\left(t \mid c ;\left\{P_{k}\right\}\right)=t^{\Delta_{0}-3 P_{v}^{2}+2 P_{\bullet} P_{v}} e^{\frac{P_{v} t^{2}}{2}}\left[1+\sum_{n=1}^{\infty} \mathscr{G}_{n}\left(c ;\left\{P_{k}\right\}\right) t^{-2 n}\right] .
$$


Algebraic definition 2.33 determines the exponent $\tilde{\alpha}=\Delta_{0}-3 P_{v}^{2}+2 P \cdot P_{v}$ in the above and also allows to compute the coefficients $\mathscr{G}_{n}$. One has, in particular,

$$
\begin{aligned}
\mathscr{G}_{1}\left(c ;\left\{P_{k}\right\}\right) & =6 P_{v}^{3}-6 P \cdot P_{v}^{2}+\left(P_{\bullet}^{2}-3 \Delta_{0}-\frac{c-1}{8}\right) P_{v}+2 P_{\bullet} \Delta_{0}, \\
\mathscr{G}_{2}\left(c ;\left\{P_{k}\right\}\right) & =\frac{1}{2} \mathscr{G}_{1}\left(c ;\left\{P_{k}\right\}\right)^{2}+\frac{105}{4} P_{v}^{4}-35 P \cdot P_{v}^{3}+\left(12 P_{\bullet}^{2}-\frac{33}{2} \Delta_{0}-\frac{13 c-19}{8}\right) P_{v}^{2}+ \\
& +\left(-P_{\bullet}^{2}+18 \Delta_{0}+\frac{19 c-31}{24}\right) P_{\bullet} P_{v}+\frac{1}{4}\left(-16 P_{\bullet}^{2}+\Delta_{0}+c-2\right) \Delta_{0} .
\end{aligned}
$$

Conjecture 2.2. Confluent conformal blocks of type $\mathscr{G}$ exponentiate as

$$
\mathscr{G}\left(t \sqrt{\frac{2 i}{b}} \mid c ; \frac{i \theta_{0}}{b}, \frac{i \theta_{\bullet}}{b}, \frac{i v}{b}\right) \stackrel{b \rightarrow 0}{\sim} \text { const } \cdot \exp \left\{b^{-2} \tilde{\mathscr{U}}\left(t \mid \theta_{0}, \theta_{\bullet}, v\right)\right\},
$$

with

$$
\tilde{\mathscr{U}}\left(t \mid \theta_{0}, \theta_{\bullet}, v\right)=-v t^{2}+\left(\delta_{0}+3 v^{2}-2 \theta_{\bullet} v\right) \ln t+\sum_{n=1}^{\infty} \tilde{\mathscr{U}}_{n}\left(\theta_{0}, \theta_{\bullet}, v\right) t^{-2 n} .
$$

Let record the first coefficients of the classical CB of type $\mathscr{G}$ :

$$
\begin{aligned}
& \tilde{\mathscr{U}}_{1}\left(\theta_{0}, \theta_{\bullet}, v\right)=-\frac{1}{2}\left[6 v^{3}-6 \theta_{\bullet} v^{2}+\left(\theta_{\bullet}^{2}+3 \delta_{0}+\frac{3}{4}\right) v-2 \delta_{0} \theta \cdot\right], \\
& \tilde{\mathscr{U}}_{2}\left(\theta_{0}, \theta_{\bullet}, v\right)=-\frac{1}{4}\left[\frac{105}{4} v^{4}-35 \theta_{\bullet} v^{3}+\left(12 \theta_{\bullet}^{2}+\frac{33}{2} \delta_{0}+\frac{39}{4}\right) v^{2}-\left(\theta_{\bullet}^{2}+18 \delta_{0}+\frac{19}{4}\right) \theta_{\bullet} v+\left(4 \theta_{\bullet}^{2}+\frac{1}{4} \delta_{0}+\frac{3}{2}\right) \delta_{0}\right] .
\end{aligned}
$$

In the next section, they will be compared with the coefficients of the strong-coupling expansion of accessory parameter function of the biconfluent Heun equation.

\section{Accessory parameter functions of Bohr-Sommerfeld type}

\subsection{Bohr-Sommerfeld periods}

Consider the Schroedinger equation

$$
\hbar^{2} \psi^{\prime \prime}(z)=U(z) \psi(z),
$$

with a rational potential $U(z)$ which can also analytically depend on $\hbar$, so that $U(z)=\sum_{n=0}^{\infty} \hbar^{n} U_{n}(z)$ with $U_{0}(z) \neq 0$. It admits a formal WKB solution

$$
\psi(z)=\exp \left\{\sum_{n=-1}^{\infty} \hbar^{n} \int^{z} S_{n}(z) d z\right\},
$$

where $S_{-1}(z) \equiv p(z)=\sqrt{U(z)}$ and all other $S_{k}$ 's are determined by the recurrence relations

$$
S_{n}^{\prime}(z)+\sum_{k=-1}^{n+1} S_{k}(z) S_{n-k}(z)=0, \quad n \geq-1 .
$$

In particular, one has

$$
\begin{array}{ll}
S_{0}=\left(-\frac{1}{2} \ln p\right)^{\prime}, & S_{1}=\frac{2 p p^{\prime \prime}-3\left(p^{\prime}\right)^{2}}{8 p^{3}} \\
S_{2}=\left(\frac{3\left(p^{\prime}\right)^{2}}{16 p^{4}}-\frac{p^{\prime \prime}}{8 p^{3}}\right)^{\prime}, & S_{3}=-\frac{297\left(p^{\prime}\right)^{4}-396 p\left(p^{\prime}\right)^{2} p^{\prime \prime}+52 p^{2}\left(p^{\prime \prime}\right)^{2}+80 p^{2} p^{\prime} p^{\prime \prime \prime}-8 p^{3} p^{\prime \prime \prime \prime}}{128 p^{7}}
\end{array}
$$

and so on. All even contributions $S_{2 n}(z)$ are given by total derivatives. In fact, denoting

$$
S_{\text {odd }}(z):=\sum_{n=0}^{\infty} \hbar^{2 n-1} S_{2 n-1}(z), \quad S_{\text {even }}(z):=\sum_{n=0}^{\infty} \hbar^{2 n} S_{2 n}(z),
$$

it can be shown that $S_{\text {odd }}^{\prime}+2 S_{\text {even }} S_{\text {odd }}=0$, and therefore $\psi(z)=\frac{1}{\sqrt{S_{\text {odd }}(z)}} \exp \int^{z} S_{\text {odd }}(z) d z$. 
Given a cycle $\gamma$ on the Riemann surface $p^{2}=U(z)$, one may then introduce the Bohr-Sommerfeld (BS) period, describing a formal monodromy of $\psi(z)$ along $\gamma$ :

$$
v:=\frac{1}{2 \pi i} \oint_{\gamma} S_{\text {odd }}(z) d z=\sum_{n=0}^{\infty} \hbar^{2 n-1} v_{2 n-1},
$$

where we denote $v_{2 n-1}:=\frac{1}{2 \pi i} \oint_{\gamma} S_{2 n-1}(z) d z$.

\subsection{Confluent Heun}

Let us now consider the potential of $\mathrm{H}_{\mathrm{V}}$,

$$
V(z)=-\frac{\delta_{0}}{z^{2}}-\frac{\delta_{t}}{(z-t)^{2}}+\frac{1}{4}+\frac{\theta_{*}}{z}-\frac{\mathscr{E}}{z(z-t)}=\frac{P_{4}(z)}{z^{2}(z-t)^{2}}
$$

and explain how a small parameter playing the role of $\hbar$ appears in our setup. Suppose that $t$ is large and that $\mathscr{E} / t=\kappa+o(1)$. It is not difficult to understand that in this case two roots $z_{1,2}$ of the 4 th degree polynomial $P_{4}(z)$ are of order $O(1)$ and two other roots $z_{3,4}$ are close to $t$. The former two turning points are asymptotically close to the roots of $-\frac{\delta_{0}}{z^{2}}+\frac{1}{4}+\frac{\theta_{*}+\kappa}{z}=0$, the latter are asymptotic to $t+\xi$, where $\xi$ satisfies $-\frac{\delta_{t}}{\xi^{2}}+\frac{1}{4}-\frac{\kappa}{\xi}=0$. To make the differentials $S_{n}(z) d z$ single-valued on $\mathbb{C P}^{1}$, we introduce two branch cuts: one connecting $z_{1}$ and $z_{2}$ and another connecting $z_{3}$ and $z_{4}$.

Choose the cycle $\gamma$ to be the circle of radius $\sqrt{t}$, so that the branch points $z=z_{1,2}$ (and eventually the pole $z=0$ ) are inside $\gamma$, while the branch points $z=z_{3,4}$ (and eventually the poles $z=t, \infty$ ) are outside. Equivalently, we could set from the very beginning $z=\lambda \sqrt{t}$, so that in the $\lambda$-plane the first two branch points would become $\lambda_{1,2}=O\left(\frac{1}{\sqrt{t}}\right)$ and two others $\lambda_{3,4}=O(\sqrt{t})$. The cycle $\gamma$ maps to the unit circle $|\lambda|=1$ and the potential becomes $t V(\lambda \sqrt{t})=\hbar^{-2} U(\lambda)$, with $\hbar=\frac{1}{\sqrt{t}}$ and

$$
U(\lambda)=\frac{1}{4}+\frac{\hbar \theta_{*}}{\lambda}+\frac{\hbar^{3} \mathscr{E}}{\lambda(1-\hbar \lambda)}-\frac{\hbar^{2} \delta_{0}}{\lambda^{2}}-\frac{\hbar^{4} \delta_{t}}{(1-\hbar \lambda)^{2}} .
$$

Recall that $\hbar^{2} \mathscr{E}=\kappa+o(1)$, and therefore $U(\lambda)=\frac{1}{4}+O(\hbar)$. In order to calculate different contributions to the BS period $v$, it now suffices to expand the integrands in $\hbar$ and compute the residue at 0 or $\infty$. Substituting

$$
\mathscr{E}=\kappa t+\sum_{n=0}^{\infty} \mathscr{E}_{n} t^{-n}
$$

we find that

$$
\begin{aligned}
v_{-1}= & \frac{1}{2 \pi i} \oint_{|\lambda|=1} p(\lambda) d \lambda=\left(\kappa+\theta_{*}\right) \hbar+\left(\mathscr{E}_{0}-2 \kappa^{2}-2 \kappa \theta_{*}\right) \hbar^{3}+ \\
& +\left[\mathscr{E}_{1}-2 \mathscr{E}_{0}\left(\theta_{*}+2 \kappa\right)+2 \kappa\left(6 \kappa^{2}+3 \theta_{*}^{2}+9 \kappa \theta_{*}+\delta_{0}+\delta_{t}\right)+2 \delta_{t} \theta_{*}\right] \hbar^{5}+ \\
& +\left[\mathscr{E}_{2}-2 \mathscr{E}_{1}\left(\theta_{*}+2 \kappa\right)+2 \mathscr{E}_{0}\left(-\mathscr{E}_{0}+\delta_{0}+\delta_{t}+3 \theta_{*}^{2}+18 \kappa \theta_{*}+18 \kappa^{2}\right)-100 \kappa^{2}\left(\theta_{*}+\kappa\right)^{2}\right. \\
& \left.\quad-12\left(\theta_{*}+2 \kappa\right)\left(\kappa \delta_{0}+\kappa \delta_{t}+\delta_{t} \theta_{*}\right)-20 \theta_{*}^{2} \kappa\left(\theta_{*}+\kappa\right)-4 \delta_{0} \delta_{t}\right] \hbar^{7}+O\left(\hbar^{9}\right), \\
v_{1}= & \frac{1}{2 \pi i} \oint_{|\lambda|=1} \frac{2 p p^{\prime \prime}-3\left(p^{\prime}\right)^{2}}{8 p^{3}} d \lambda=-4 \kappa\left(\kappa+\theta_{*}\right) \hbar^{5}+O\left(\hbar^{7}\right), \\
v_{3}= & -48 \kappa\left(\kappa+\theta_{*}\right) \hbar^{7}+O\left(\hbar^{9}\right), \quad \ldots
\end{aligned}
$$

Note in particular that the leading contribution to the period is of 0th order: $v=\kappa+\theta_{*}+O(\hbar)$.

Inverting the relation $v=v(\mathscr{E}, t)$ to express $\mathscr{E}=\mathscr{E}^{[\mathrm{BS}]}(t \mid v)$ as a function of $v$ and $t$ is equivalent to asking all quantum ( $\hbar$-dependent) corrections to the BS period to vanish. It follows that

$$
\mathscr{E}_{0}=2 \kappa\left(\kappa+\theta_{*}\right)=2\left(v-\theta_{*}\right) v, \quad \mathscr{E}_{1}=-\mathscr{U}_{1}\left(\theta_{0}, \theta_{t}, \theta_{*}, v\right), \quad \mathscr{E}_{2}=-2 \mathscr{U}_{2}\left(\theta_{0}, \theta_{t}, \theta_{*}, v\right), \quad \ldots,
$$

where $\mathscr{U}_{1,2}$ coincide with those given in 2.31. It now becomes straightforward to formulate Conjecture B for confluent CBs of type $\mathscr{D}$. 
Conjecture 3.1. Let $v$ be the Bohr-Sommerfeld period along the cycle described above. Then

$$
\mathscr{E}^{[\mathrm{BS}]}\left(t \mid \theta_{0}, \theta_{t}, \theta_{*}, v\right)=t \frac{\partial}{\partial t} \mathscr{U}_{N_{f}=3}\left(t \mid \theta_{0}, \theta_{t}, \theta_{*}, v\right)
$$

where $\mathscr{U}_{N_{f}=3}\left(t \mid \theta_{0}, \theta_{t}, \theta_{*}, v\right)$ is the classical confluent conformal block of type $\mathscr{D}$.

\subsection{Biconfluent Heun}

Let us now follow a similar approach for the biconfluent HE. The potential of $\mathrm{H}_{\mathrm{IV}}$ is given by

$$
V(z)=-\frac{\delta_{0}}{z^{2}}-\frac{\mathscr{E}}{z}+2 \theta_{\bullet}+(z+t)^{2}=\frac{Q_{4}(z)}{z^{2}}
$$

Assume again that $t$ is large and that $\mathscr{E} / t=\kappa+o(1)$. Two zeros $z_{1,2}$ of $Q_{4}(z)$ are asymptotic to $\frac{\xi_{1,2}}{t}$, where $\xi_{1,2}$ satisfy the equation $-\frac{\delta_{0}}{\xi^{2}}-\frac{\kappa}{\xi}+1=0$. Two other zeros $z_{3,4}$ are asymptotic to $-t+\xi_{3,4}$, where $\xi_{3,4}$ satisfy the equation $\kappa+2 \theta_{\bullet}+\xi^{2}=0$. Introducing branch cuts going from $z_{1}$ to $z_{2}$ and from $z_{3}$ to $z_{4}$, we choose $\gamma$ defining the BS period to be the unit circle. Also, we rewrite the potential as $V(z)=\hbar^{-2} U(z)$, with $\hbar=t^{-1}$ and

$$
U(z)=1+2 \hbar z-\frac{\hbar^{2} \mathscr{E}}{z}+\hbar^{2}\left(z^{2}-\frac{\delta_{0}}{z^{2}}+2 \theta\right)
$$

By the above assumption, $\hbar \mathscr{E}=\kappa+o(1)$. Making the ansatz [3.9], one obtains

$$
\begin{aligned}
v_{-1}= & -\frac{1}{2} \kappa \hbar-\frac{1}{2} \mathscr{E}_{0} \hbar^{2}+\frac{1}{2}\left(-\mathscr{E}_{1}+\delta_{0}+\kappa \theta \cdot+\frac{3}{4} \kappa^{2}\right) \hbar^{3}+\frac{1}{4}\left(-2 \mathscr{E}_{2}+\left(2 \theta_{\bullet}+3 \kappa\right) \mathscr{E}_{0}\right) \hbar^{4}+ \\
& +\frac{1}{4}\left(-2 \mathscr{E}_{3}+\mathscr{E}_{1}\left(2 \theta_{\bullet}+3 \kappa\right)+\frac{3}{2} \mathscr{E}_{0}^{2}-\frac{15}{4} \kappa^{2}\left(2 \theta_{\bullet}+\kappa\right)-6 \delta_{0}\left(\theta_{\bullet}+\kappa\right)-3 \theta_{\bullet}^{2} \kappa\right) \hbar^{5}+O\left(\hbar^{6}\right), \\
v_{1}= & -\frac{3}{16} \kappa \hbar^{3}-\frac{3}{16} \mathscr{E}_{0} \hbar^{4}+\frac{1}{64}\left(-12 \mathscr{E}_{1}+60 \delta_{0}+100 \theta_{\bullet} \kappa+105 \kappa^{2}\right) \hbar^{5}+O\left(\hbar^{6}\right), \\
v_{3}= & -\frac{315}{256} \kappa \hbar^{5}+O\left(\hbar^{6}\right), \quad \ldots
\end{aligned}
$$

Requiring the quantum corrections to the BS period to vanish, we find that

$$
\begin{aligned}
\mathscr{E}_{0}=\mathscr{E}_{2} & =\mathscr{E}_{4}=\ldots=0, \quad \kappa=-2 v, \quad \mathscr{E}_{1}=\delta_{0}+3 v^{2}-2 \theta \bullet v, \\
\mathscr{E}_{3} & =6 v^{3}-6 \theta \cdot v^{2}+\left(\theta_{\bullet}^{2}+3 \delta_{0}+\frac{3}{4}\right) v-2 \delta_{0} \theta ., \quad \ldots
\end{aligned}
$$

Compraison of these expressions with 2.37-2.38 finally leads to a variant of Conjecture B for confluent CBs of type $\mathscr{G}$.

Conjecture 3.2. Let $v$ be the Bohr-Sommerfeld period along the cycle described above. Then

$$
\mathscr{E}^{[\mathrm{BS}]}\left(t \mid \theta_{0}, \theta_{\bullet}, v\right)=\frac{\partial}{\partial t} \tilde{\mathscr{U}}\left(t \mid \theta_{0}, \theta_{\bullet}, v\right)
$$

where $\tilde{\mathscr{U}}\left(t \mid \theta_{0}, \theta_{\bullet}, v\right)$ denotes the classical confluent conformal block of type $\mathscr{G}$.

\section{Discussion}

We conclude by mentioning a few questions that have been left outside the scope of this note.

- All-order Bohr-Sommerfeld relation used to define the accessory parameter functions is an analog of the Hill determinant evaluation of the Mathieu characteristic exponent. Although $\mathscr{E}^{[\mathrm{BS}]}(t)$ was so far defined only as a formal asymptotic series, it can be promoted to an actual function of $t$ by relating the resummed BS period to genuine monodromy/Stokes data with the help of exact WKB techniques, see e.g. [IN AJJRT]. Classical irregular conformal blocks may then be interpreted as generating functions of canonical transformations between $(\mathscr{E}, t)$ and a pair of suitable coordinates on confluent Heun monodromy manifolds. 
- It would be interesting to identify conformal blocks whose quasiclassical limit describes accessory parameters of the confluent Heun equations other than $\mathrm{H}_{V}$ and $\mathrm{H}_{\mathrm{IV}}$. A recent work [Na2] contains a few promising candidates for this role for $\mathrm{H}_{\mathrm{III}}$ and $\mathrm{H}_{\mathrm{II}}$ (see also $\left[\mathrm{NU}\right.$ ). Even in the case of $\mathrm{H}_{\mathrm{V}}$ and $\mathrm{H}_{\mathrm{IV}}$, there exist other possibilities to define BS accessory parameter function which lead to ansätze different from [3.9]. On the gauge theory side, they correspond to different strongly coupled expansion points. An algebraic construction of the corresponding CBs of the 2nd kind does not seem to be known.

- There is a well-known correspondence between Heun and Painlevé equations under which Heun couplings $t$ are mapped to special points of Painlevé functions. It was realized in [NC, CCN, LN] that this relation can be used to compute asymptotic expansions of Heun accessory parameters. In the terminology of the present paper, $|\mathrm{NC}, \mathrm{CCN}, \mathrm{LN}|$ deal with the weak coupling expansions of Floquet characteristics of $\mathrm{H}_{\mathrm{VI}}$ and $\mathrm{H}_{\mathrm{V}}$ which can in fact be derived more easily as explained in Appendices $\mathrm{A}$ and $\mathrm{B}$ However the method of loc. cit. can be extended to strong coupling using the long-distance Painlevé asymptotic expansions from BLMST; this was effectively done for Mathieu equation $\mathrm{H}_{\mathrm{III}_{3}}$ in GMS. The Heun/Painlevé correspondence in the latter case admits an interpretation in terms of the quasiclassical limit of the Nakajima-Yoshioka blowup equations for pure SU(2) gauge theory [GG, whose generalizations to the regular case were recently studied in JN Nek2]. It should be possible to obtain (and perhaps even prove!) similar identities (implicitly present in the approach of [NC $[\mathrm{CCN}, \mathrm{LN}]$ ) relating BS accessory parameters and Painlevé functions in other cases, including those corresponding to Argyres-Douglas theories. We plan to return to these questions in a future work.

Acknowledgements. The authors are grateful to Pavlo Gavrylenko and Nikolai Iorgov for illuminating discussions, and to Hajime Nagoya for sharing a Mathematica code computing higher order contributions to confluent conformal blocks of type $\mathscr{G}$.

\section{A Heun accessory parameter from continued fractions}

It will be convenient for us here to transform the Heun equation [1.1 into its canonical form by the substitution

$$
\psi(z)=z^{\frac{1}{2}-\theta_{0}}(z-t)^{\frac{1}{2}-\theta_{t}}(z-1)^{\frac{1}{2}-\theta_{1}} \phi(z) .
$$

The resulting equation for $\phi(z)$ is given by

$$
\phi^{\prime \prime}(z)+\left(\frac{\gamma}{z}+\frac{\delta}{z-1}+\frac{\epsilon}{z-t}\right) \phi^{\prime}(z)+\frac{\alpha \beta z-q}{z(z-1)(z-t)} \phi(z)=0
$$

with

$$
\begin{gathered}
\alpha=1-\theta_{0}-\theta_{1}-\theta_{t}-\theta_{\infty}, \quad \beta=1-\theta_{0}-\theta_{1}-\theta_{t}+\theta_{\infty}, \\
\gamma=1-2 \theta_{0}, \quad \delta=1-2 \theta_{1}, \quad \epsilon=1-2 \theta_{t}, \\
q=(1-t) \mathscr{E}+\frac{\gamma \epsilon+2 \alpha \beta t-(\gamma+\delta) \epsilon t}{2} .
\end{gathered}
$$

Obviously, we have a relation $\alpha+\beta+1=\gamma+\delta+\epsilon$. In spite of loosing a symmetry $\theta_{k} \mapsto-\theta_{k}(k=0,1, t, \infty)$ present in the normal form [1.1, the canonical form turns out to be better adapted for perturbative calculation below. A similar and very much related phenomenon is known from the AGT correspondence: 4D instanton partition functions have simpler series reperesentations than 2D conformal blocks but loose certain of their manifest symmetries because of the presence of the so-called $U(1)$ factor.

Choose a basis in the space of solutions of $A .2$ which diagonalizes the composite monodromy around the pair of singular points $0, t$. Assuming that $0<|t|<1$, its elements can be represented inside the annulus $|t|<|z|<1$ in the Floquet form

$$
\phi(z)=\sum_{n \in \mathbb{Z}} c_{n} z^{n+\omega}
$$

Substituting this series into A.2], one obtains a linear 3-term recurrence relation for the coefficients,

$$
A_{n} c_{n-1}-B_{n} c_{n}+C_{n} t c_{n+1}=0
$$


where

$$
\begin{aligned}
& A_{n}=(\omega+n-1+\alpha)(\omega+n-1+\beta), \\
& B_{n}=q+(\omega+n)(\epsilon+\delta t+(\omega+n-1+\gamma)(1+t)), \\
& C_{n}=(\omega+n+1)(\omega+n+\gamma) .
\end{aligned}
$$

The recurrence relation above is the main reason we started with [A.2) instead of 1.1]. As we will see in a moment, the crucial point for the computation of the small $t$ expansion of the accessory parameter,

$$
q(t)=\sum_{n=0}^{\infty} q_{n} t^{n}
$$

is how the time $t$ appears in A.5.

For $n \geq 0$, define $u_{n}=\frac{c_{n+1}}{c_{n}}$. This change of variables transforms A.5 into a nonlinear 2-term "Riccati" equation $u_{n-1}=\frac{A_{n}}{B_{n}-t C_{n} u_{n}}$. Under assumption that $B_{n}=O(1)$ as $t \rightarrow 0$ for $n \geq 1$, one can then express $u_{0}$ as an infinite continued fraction

$$
u_{0}=\frac{A_{1}}{B_{1}-\frac{t C_{1} A_{2}}{B_{2}-\frac{t C_{2} A_{3}}{B_{3}-\ldots}} .}
$$

For $n \leq 0$, we first define rescaled coefficients $d_{-n}=c_{n} t^{n}$ and rewrite the 3-term relation A.5 as

$$
t A_{-n} d_{n+1}-B_{-n} d_{n}+C_{-n} d_{n-1}=0,
$$

Introducing $v_{n}=\frac{d_{n+1}}{d_{n}}$, we have $v_{n-1}=\frac{C_{-n}}{B_{-n}-t A_{-n} \nu_{n}}$, so that

$$
v_{0}=\frac{C_{-1}}{B_{-1}-\frac{t A_{-1} C_{-2}}{B_{-2}-\frac{t A_{-2} C_{-3}}{B_{-3} \cdots}}},
$$

under assumption that $B_{n}=O(1)$ as $t \rightarrow 0$ for $n \leq-1$. From $A_{0} c_{-1}-B_{0} c_{0}+t C_{0} c_{1}=0$ it follows that $t\left(C_{0} u_{0}+A_{0} v_{0}\right)=B_{0}$, which ultimately gives an equation determining the accessory parameter $q$ as a function of $t$ for given $\omega$ :

$$
\frac{t C_{0} A_{1}}{B_{1}-\frac{t C_{1} A_{2}}{B_{2}-\frac{t C_{2} A_{3}}{B_{3}-\ldots}}}+\frac{t A_{0} C_{-1}}{B_{-1}-\frac{t A_{-1} C_{-2}}{B_{-2}-\frac{t A_{-2} C_{-3}}{B_{-}-\ldots}}}=B_{0} .
$$

Note that $\left\{A_{n}\right\}$ and $\left\{C_{n}\right\}$ are just some monodromy-dependent constants; thus $q$ enters into A.11] only through $\left\{B_{n}\right\}$. Substituting into A.6b) the expansion (A.7), the coefficients $q_{k}$ can be recursively determined from (A.11] by truncating the continued fraction ladder at the desired order in $t$.

For example, at order $O(1)$ we have $B_{0}=O(t)$, and therefore

$$
q_{0}=-\omega(\omega+\gamma+\epsilon-1) .
$$

At order $O(t)$, one has $B_{0}=t\left(\frac{C_{0} A_{1}}{B_{1}}+\frac{A_{0} C_{-1}}{B_{-1}}\right)+O\left(t^{2}\right)$, which gives

$$
q_{1}=-\omega(\omega+\gamma+\epsilon-1)+\frac{(\omega+1)(\omega+\alpha)(\omega+\beta)(\omega+\gamma)}{2 \omega+\gamma+\epsilon}-\frac{\omega(\omega+\alpha-1)(\omega+\beta-1)(\omega+\gamma-1)}{2 \omega+\gamma+\epsilon-2},
$$

and so on. Substituting these coefficients into $\overline{\mathrm{A} .3 \mathrm{c}}$, we obtain the expansion of $\mathscr{E}=\mathscr{E}_{\mathrm{VI}}^{[\mathrm{F}]}(t \mid \sigma)$ :

$$
\mathscr{E}_{\mathrm{VI}}^{[\mathrm{F}]}(t \mid \sigma)=\left(\delta_{\sigma}-\delta_{0}-\delta_{t}\right)+\mathscr{W}_{1}\left(\left\{\delta_{k}\right\}\right) t+2 \mathbb{W}_{2}\left(\left\{\delta_{k}\right\}\right) t^{2}+O\left(t^{3}\right),
$$

where $\mathbb{W}_{1,2}\left(\left\{\delta_{k}\right\}\right)$ are given by 2.6a)-2.6b), $\left\{\delta_{k}\right\}$ are defined by $[1.10],\left\{\theta_{k}\right\}$ are related to $\alpha, \beta, \gamma, \delta, \epsilon$ by $[$ A.3a) A.3b, and the Floquet exponent appears only in $\sigma=\omega-\theta_{0}-\theta_{t}+\frac{1}{2}$. One thus easily recognizes in (A.13) the expansion of the logarithmic derivative $t \frac{\partial}{\partial t} \mathscr{W}\left(t \mid\left\{\delta_{k}\right\}\right)$ of the classical regular conformal block 1.8 . 


\section{B Floquet characteristics for confluent Heun equations}

Throughout this section we refer to the notations of Table 1 . The perturbative computation of accessory parameter functions of Floquet type presented here allows to check confluent Conjecture B [1.12] at any desired order in $t$. Assuming the conjecture is true, this technique provides the most elementary method of computing classical CBs of the 1st kind.

\section{B.1 Equation $\mathbf{H}_{V}$}

The change of variables $\psi(z)=z^{\frac{1}{2}-\theta_{0}}(z-t)^{\frac{1}{2}-\theta_{t}} e^{\frac{z}{2}} \phi(z)$ transforms the confluent HE into the canonical form,

$$
\phi^{\prime \prime}(z)+\left(\frac{\beta}{z}+\frac{\gamma}{z-t}+1\right) \phi^{\prime}(z)+\frac{\alpha z-q}{z(z-t)} \phi(z)=0,
$$

with

$$
\begin{gathered}
\alpha=1-\theta_{0}-\theta_{t}-\theta_{*}, \quad \beta=1-2 \theta_{0}, \quad \gamma=1-2 \theta_{t}, \\
q=-\mathscr{E}+\alpha t-\frac{1}{2}(\beta+t) \gamma .
\end{gathered}
$$

Looking for the solutions of $\mathrm{B}$.1] in the Floquet form A.4], we arrive at the same continued fraction equation A.11 for $q(t)$, except that the coefficients $\left\{A_{n}\right\},\left\{B_{n}\right\},\left\{C_{n}\right\}$ are now given by

$$
\begin{aligned}
& A_{n}=\omega+n-1+\alpha, \\
& B_{n}=q-(\omega+n)(\omega+n-1+\beta+\gamma-t), \\
& C_{n}=-(\omega+n+1)(\omega+n+\beta) .
\end{aligned}
$$

The expansion of $q(t)$ can now be computed to any desired order. Its first terms read

$$
q(t)=\omega(\omega+\beta+\gamma-1)+\left[-\omega+\frac{(\omega+1)(\omega+\alpha)(\omega+\beta)}{2 \omega+\beta+\gamma}-\frac{\omega(\omega+\alpha-1)(\omega+\beta-1)}{2 \omega+\beta+\gamma-2}\right] t+O\left(t^{2}\right) .
$$

The expansion of the Floquet characteristic $\mathscr{E}$ is then obtained from B.2b (note that, in contrast with nonconfluent HE, the coefficients of expansions of $\mathscr{E}$ and $-q$ coincide starting from the quadratic term). If we denote $\sigma=\omega-\theta_{0}-\theta_{t}+\frac{1}{2}$ as before, then

$$
\begin{aligned}
\mathscr{E}_{\mathrm{V}}^{[\mathrm{F}]}(t \mid \sigma) & =\left(\delta_{\sigma}-\delta_{0}-\delta_{t}\right)-\frac{\theta_{*}\left(\delta_{\sigma}-\delta_{0}+\delta_{t}\right)}{2 \delta_{\sigma}} t+ \\
& +\left[\frac{\theta_{*}^{2}\left(\delta_{\sigma}^{2}-\left(\delta_{0}-\delta_{t}\right)^{2}\right)}{8 \delta_{\sigma}^{3}}-\frac{\left(3 \theta_{*}^{2}+\delta_{\sigma}\right)\left(\delta_{\sigma}^{2}+2 \delta_{\sigma}\left(\delta_{0}+\delta_{t}\right)-3\left(\delta_{0}-\delta_{t}\right)^{2}\right)}{8 \delta_{\sigma}^{2}\left(3+4 \delta_{\sigma}\right)}\right] t^{2}+O\left(t^{3}\right) .
\end{aligned}
$$

It is instructive to check that this indeed agrees with the limit 1.11a and reproduces the expansion of $t \frac{\partial}{\partial t} W_{N_{f}=3}(t), \operatorname{cf} 2.14$.

\section{B.2 Equation $\mathrm{H}_{\mathrm{III}_{1}}$}

After the transformation $\psi(z)=\sqrt{z} e^{\frac{z}{2}+\frac{t}{2 z}} \phi(z)$, the equation $\mathrm{H}_{\mathrm{III}_{1}}$ becomes

$$
\phi^{\prime \prime}(z)+\left(1+\frac{1}{z}-\frac{t}{z^{2}}\right) \phi^{\prime}(z)+\left(\frac{t\left(\frac{1}{2}-\theta_{\star}\right)}{z^{3}}+\frac{\mathscr{E}-\frac{t}{2}-\frac{1}{4}}{z^{2}}+\frac{\frac{1}{2}-\theta_{*}}{z}\right) \phi(z)=0 .
$$

This is not the canonical form of doubly confluent Heun equation given in http://dlmf.nist.gov/31.12.E2 which is obtained by a slightly different change of variables; yet it is more convenient for us to continue with B.6). The Floquet substitution A.4 with $\omega=\sigma$ yields the equation A.11 with

$$
A_{n}=\theta_{*}-\sigma-n+\frac{1}{2}, \quad B_{n}=\mathscr{E}-\frac{t}{2}-\frac{1}{4}+(\sigma+n)^{2}, \quad C_{n}=\theta_{\star}+\sigma+n+\frac{1}{2},
$$

which then allows to compute the small $t$ expansion of $\mathscr{E}$. Its first few terms are given by

$$
\mathscr{E}_{\mathrm{III}_{1}}^{[\mathrm{F}]}(t \mid \sigma)=\delta_{\sigma}+\frac{\theta_{*} \theta_{\star}}{2 \delta_{\sigma}} t+\left(\frac{\left(3 \theta_{*}^{2}+\delta_{\sigma}\right)\left(3 \theta_{\star}^{2}+\delta_{\sigma}\right)}{8 \delta_{\sigma}^{2}\left(3+4 \delta_{\sigma}\right)}-\frac{\theta_{*}^{2} \theta_{\star}^{2}}{8 \delta_{\sigma}^{3}}\right) t^{2}+O\left(t^{3}\right) .
$$




\section{B.3 Equation $\mathrm{H}_{\mathrm{III}_{2}}$}

In this case, the relevant change of variables is $\psi(z)=\sqrt{z} e^{\frac{z}{2}} \phi(z)$. It transforms the equation $\mathrm{H}_{\mathrm{III}_{2}}$ into

$$
\phi^{\prime \prime}(z)+\left(1+\frac{1}{z}\right) \phi^{\prime}(z)+\left(-\frac{t}{z^{3}}+\frac{\mathscr{E}-\frac{1}{4}}{z^{2}}+\frac{\frac{1}{2}-\theta_{*}}{z}\right) \phi(z)=0 .
$$

The Floquet substitution [A.4 with $\omega=\sigma$ again gives the equation A.11, whose coefficients are now given by

$$
A_{n}=\theta_{*}-\sigma-n+\frac{1}{2}, \quad B_{n}=\mathscr{E}-\frac{1}{4}+(\sigma+n)^{2}, \quad C_{n}=1,
$$

The expansion of accessory parameter function reads

$$
\mathscr{E}_{\mathrm{III}_{2}}^{[\mathrm{F}]}(t \mid \sigma)=\delta_{\sigma}+\frac{\theta_{*}}{2 \delta_{\sigma}} t+\frac{\left(5 \delta_{\sigma}-3\right) \theta_{*}^{2}+3 \delta_{\sigma}^{2}}{8 \delta_{\sigma}^{3}\left(3+4 \delta_{\sigma}\right)} t^{2}+\frac{\theta_{*}\left(\left(7 \delta_{\sigma}-6\right) \delta_{\sigma}^{2}+\left(9 \delta_{\sigma}^{2}-19 \delta_{\sigma}+6\right) \theta_{*}^{2}\right)}{16 \delta_{\sigma}^{5}\left(3+4 \delta_{\sigma}\right)\left(2+\delta_{\sigma}\right)} t^{3}+O\left(t^{4}\right) .
$$

\section{B.4 Equation $\mathrm{H}_{\mathrm{III}_{3}}$}

In this last case (recall that it is equivalent to Mathieu equation) the analog of the above calculations is rather well-known. There is no need to transform the equation. Making the Floquet ansatz $\psi(z)=\sum_{n \in \mathbb{Z}} c_{n} z^{\frac{1}{2}+\sigma+n}$ directly in the normal form, we arrive at a 3-term recurrence relation and equation A.11] for $\mathscr{E}$ with

$$
A_{n}=C_{n}=1, \quad B_{n}=\mathscr{E}-\frac{1}{4}+(\sigma+n)^{2},
$$

This gives the expansion of the Mathieu characteristic value:

$$
\mathscr{E}_{\mathrm{III}_{3}}^{[\mathrm{F}]}(t \mid \sigma)=\delta_{\sigma}+\frac{t}{2 \delta_{\sigma}}+\frac{5 \delta_{\sigma}-3}{8 \delta_{\sigma}^{3}\left(3+4 \delta_{\sigma}\right)} t^{2}+\frac{9 \delta_{\sigma}^{2}-19 \delta_{\sigma}+6}{16 \delta_{\sigma}^{5}\left(3+4 \delta_{\sigma}\right)\left(2+\delta_{\sigma}\right)} t^{3}+O\left(t^{4}\right) .
$$

To facilitate comparison with the literature, note that the parameters $v$ and $q$ in e.g. [AS, Eq. 20.3.15] and DLMF Eq. 28.15.E1 correspond to our $2 \sigma$ and $4 \sqrt{t}$ so that $\delta_{\sigma}=\frac{1-v^{2}}{4}$.

\section{References}

[AS] M. Abramowitz, I. Stegun, Handbook of Mathematical Functions, Dover, (1964).

[AFLT] V. A. Alba, V. A. Fateev, A. V. Litvinov, G. M. Tarnopolsky, On combinatorial expansion of the conformal blocks arising from AGT conjecture, Lett. Math. Phys. 98, (2011), 33-64; arXiv:1012.1312 [hep-th].

[AGT] L. F. Alday, D. Gaiotto, Y. Tachikawa, Liouville correlation functions from four-dimensional gauge theories, Lett. Math. Phys. 91, (2010), 167-197; arXiv:0906.3219 [hep-th].

[AJJRT] S. K. Ashok, D. P. Jatkar, R. R. John, M. Raman, J. Troost, Exact WKB analysis of $\mathscr{N}=2$ gauge theories, J. High Energ. Phys. 2016, (2016), 115; arXiv:1604.05520 [hep-th].

[BD] G. Başar, G. V. Dunne, Resurgence and the Nekrasov-Shatashvili limit: connecting weak and strong coupling in the Mathieu and Lamé systems, JHEP 1502, (2015), 160; arXiv:1501.05671 [hep-th].

[BPZ] A. A. Belavin, A. M. Polyakov, A. B. Zamolodchikov, Infinite conformal symmetry in two-dimensional quantum field theory, Nucl. Phys. B241, (1984), 333-380.

[BDK] M. Beşken, S. Datta, P. Kraus, Semi-classical Virasoro blocks: proof of exponentiation, arXiv:1910.04169v1 [hep-th].

[BPM] J. Blandin, R. Pons, G. Marcilhacy, General solution of Teukolsky's equation, Lett. Nuovo Cimento 38, (1983), 561-567. 
[BMT] G. Bonelli, K. Maruyoshi, A. Tanzini, Wild quiver gauge theories, J. High Energ. Phys. 2012:31, (2012); arXiv:1112.1691 [hep-th].

[BLMST] G. Bonelli, O. Lisovyy, K. Maruyoshi, A. Sciarappa, A. Tanzini, On Painlevé/gauge theory correspondence, Lett. Math. Phys. 107, (2017), 2359-2413; arXiv:1612.06235v1 [hep-th].

[CCC] B. Carneiro da Cunha, J. P. Cavalcante, Confluent conformal blocks and the Teukolsky master equation, Phys. Rev. D102, (2020), 105013; arXiv:1906.10638 [hep-th].

[CCR] B. Carneiro da Cunha, M. Carvalho de Almeida, A. Rabelo de Queiroz, On the existence of monodromies for the Rabi model, J. Phys. A49, (2016), 194002; arXiv:1508.01342 [math-ph].

[CCN] B. Carneiro da Cunha, F. Novaes, Kerr scattering Coefficients via isomonodromy, J. High Energ. Phys. 2015, 144 (2015); arXiv:1506.06588 [hep-th].

[CM] L. Chekhov, M. Mazzocco, Colliding holes in Riemann surfaces and quantum cluster algebras, Nonlinearity 31, (2018), 54; arXiv:1509.07044 [math-ph].

[CMR] L. Chekhov, M. Mazzocco, V. Rubtsov, Painlevé monodromy manifolds, decorated character varieties and cluster algebras, Int. Math. Res. Not. 2017, (2017), 7639-7691; arXiv:1511.03851v1 [math-ph].

[DLMF] NIST Digital Library of Mathematical Functions, http://dlmf.nist.gov/

[DK] B. Dubrovin, A. Kapaev, A Riemann-Hilbert approach to the Heun equation, SIGMA 14, (2018), 093; arXiv:1809.02311v1 [math.CA].

[Fiz] P. Fiziev, Exact solutions of Regge-Wheeler equation and quasi-normal modes of compact objects, Class.Quant.Grav. 23, (2006), 2447-2468; arXiv:gr-qc/0509123.

[FIKN] A. S. Fokas, A. R. Its, A. A. Kapaev, V. Yu. Novokshenov, Painlevé transcendents: the Riemann-Hilbert approach, Mathematical Surveys and Monographs 128, AMS, Providence, RI, (2006).

[Fuchs] R. Fuchs, Sur quelques équations différentielles linéaires du second ordre, C. R. Acad. Sci. Paris 141, (1905), 555-558.

[Gai] D. Gaiotto, Asymptotically free $\mathscr{N}=2$ theories and irregular conformal blocks, arXiv:0908.0307 [hep-th].

[GT] D. Gaiotto, J. Teschner, Irregular singularities in Liouville theory and Argyres-Douglas type gauge theories, I, J. High Energ. Phys. 2012, 50 (2012); arXiv:1203.1052 [hep-th].

[GMS] P. Gavrylenko, A. Marshakov, A. Stoyan, Irregular conformal blocks, Painlevé III and the blow-up equations, arXiv:2006.15652 [math-ph].

[Gar] R. Garnier, Sur des équations différentielles du troisième ordre dont l'intégrale générale est uniforme et sur une classe d'équations nouvelles d'ordre supérieur dont l'intégrale générale a ses points critiques fixes, Ann. Sci. de l'É.N.S. 29, (1912), 1-126.

[GG] A. Grassi, J. Gu, BPS relations from spectral problems and blowup equations, Lett. Math. Phys. 109, (2019), 1271-1302; arXiv:1609.05914 [hep-th].

[GGM] A. Grassi, J. Gu, M. Mariño, Non-perturbative approaches to the quantum Seiberg-Witten curve, J. High Energ. Phys. 2020, (2020), 106; arXiv:1908.07065 [hep-th].

[IN] K. Iwaki, T. Nakanishi, Exact WKB analysis and cluster algebras, J. Phys. A47, (2014), 474009; arXiv:1401.7094 [math.CA].

[JN] S. Jeong, N. Nekrasov, Riemann-Hilbert correspondence and blown up surface defects, J. High Energ. Phys. 2020, (2020), 6; arXiv:2007.03660 [hep-th].

[HM] W. He, Y.-G. Miao, Magnetic expansion of Nekrasov theory: the SU(2) pure gauge theory, Phys. Rev. D82, (2010), 025020; arXiv:1006.1214v3 [hep-th]. 
[Hei] E. Heine, Hanbuch Der Kugelfunktionen, Vol. 2, Georg Reimer, Berlin, (1878), p.81.

[HK] L. Hollands and O. Kidwai, Higher length-twist coordinates, generalized Heun's opers, and twisted superpotentials, Adv. Theor. Math. Phys. 22, (2018), 1713-1822; arXiv:1710.04438 [hep-th].

[Lea] E. W. Leaver, Solutions to a generalized spheroidal wave equation: Teukolsky's equations in general relativity, and the two-center problem in molecular quantum mechanics, J. Math. Phys. 27, (1986), 1238.

[LN] M. Lencsés, F. Novaes, Classical conformal blocks and accessory parameters from isomonodromic deformations, JHEP 04, (2018), 096; arXiv:1709.03476 [hep-th].

[LNR] O. Lisovyy, H. Nagoya, J. Roussillon, Irregular conformal blocks and connection formulae for Painlevé V functions, J. Math. Phys. 59, (2018), 091409; arXiv:1806.08344 [math-ph].

[LLNZ] A. Litvinov, S. Lukyanov, N. Nekrasov, A. Zamolodchikov, Classical conformal blocks and Painlevé VI, J. High Energy Phys. (2014) 2014: 144; arXiv:1309.4700 [hep-th].

[MPS] A. J. Maciejewski, M. Przybylska, T. Stachowiak, Full spectrum of the Rabi model, Phys. Lett. A378, (2014), 16-20; arXiv:1310.8655v1 [math-ph].

[MM] A. Mironov, A. Morozov, Nekrasov Functions and exact Bohr-Sommerfeld integrals, J. High Energ. Phys. 2010, 40 (2010); arXiv:0910.5670v2 [hep-th].

[Men] P. Menotti, On the monodromy problem for the four-punctured sphere, J. Phys. A47, (2014), 415201; arXiv:1401.2409 [hep-th].

[Na1] H. Nagoya, Irregular conformal blocks, with an application to the fifth and fourth Painleve equations, J. Math. Phys. 56, (2015), 123505; arXiv:1505.02398v3 [math-ph].

[Na2] H. Nagoya, Remarks on irregular conformal blocks and Painlevé III and II tau functions, in "Proceedings of the Meeting for Study of Number Theory, Hopf Algebras and Related Topics”; arXiv:1804.04782v2 [math-ph].

[Nek1] N. A. Nekrasov, Seiberg-Witten prepotential from instanton counting, Adv. Theor. Math. Phys. 7, (2003), 831-864; arXiv:hep-th/0206161.

[Nek2] N. A. Nekrasov, Blowups in BPS/CFT correspondence, and Painlevé VI, arXiv:2007.03646 [hep-th].

[NS] N. S. Nekrasov, S. L. Shatashvili, Quantization of integrable systems and four dimensional gauge theories, arXiv:0908.4052 [hep-th].

[NO] N. Nekrasov, A. Okounkov, Seiberg-Witten theory and random partitions, in "The unity of mathematics", pp. 525-596, Progr. Math. 244, Birkhäuser Boston, Boston, MA, (2006); arXiv:hep-th/0306238.

[NU] T. Nishinaka, T. Uetoko, Argyres-Douglas theories and Liouville irregular states, J. High Energ. Phys. 2019, (2019), 104; arXiv:1905.03795 [hep-th].

[NC] F. Novaes, B. Carneiro da Cunha, Isomonodromy, Painlevé transcendents and scattering off ofblack holes, JHEP 07 (2014) 132; arXiv:1404.5188 [hep-th].

[Nov] V. Yu. Novokshenov, Movable poles of the solutions of Painleve's equation of the third kind and their relation with Mathieu functions, Func. Anal. Appl. 20, (1986), 113-123.

[PP1] M. Piatek, A. R. Pietrykowski, Classical irregular block, $\mathscr{N}=2$ pure gauge theory and Mathieu equation, J. High Energ. Phys. (2014) 2014: 32; arXiv:1407.0305v1 [hep-th].

[PP2] M. R. Piatek, A. R. Pietrykowski, Classical irregular blocks, Hill's equation and PT-symmetric periodic complex potentials, J. High Energ. Phys. 2016, 131 (2016); arXiv:1604.03574 [hep-th].

[PP3] M. Piatek, A. R. Pietrykowski, Solving Heun's equation using conformal blocks, Nucl. Phys. B938, (2019), 543-570; arXiv:1708.06135 [hep-th]. 
[RZ1] C. Rim, H. Zhang, Classical Virasoro irregular conformal block, J. High Energ. Phys. 2015, 163 (2015); arXiv:1504.07910v2 [hep-th].

[RZ2] C. Rim, H. Zhang, Classical Virasoro irregular conformal block II, J. High Energ. Phys. 2015, 97 (2015); arXiv:1506.03561v2 [hep-th].

[SL] S. Yu. Slavyanov, W. Lay, Special functions: a unified theory based on singularities, Oxford Univ. Press, (2000).

[STU] H. Suzuki, E. Takasugi, H. Umetsu, Perturbations of Kerr-de Sitter black hole and Heun's equations, Prog.Theor.Phys. 100, (1998), 491-505; arXiv:gr-qc/9805064.

[Tes] J. Teschner, Quantization of the Hitchin moduli spaces, Liouville theory, and the geometric Langlands correspondence I, Adv. Theor. Math. Phys. 15, Number 2 (2011), 471-564; arXiv:1005.2846v5 [hep-th].

[WW] E. T. Whittaker, G. N. Watson, A course in modern analysis, 4th edition, Cambridge Univ. Press, (1927).

[Zam] Al. B. Zamolodchikov, Two-dimensional conformal symmetry and critical four-spin correlation functions in the Ashkin-Teller model, Sov. Phys. JETP 63 (5), (1986), 1061-1066.

[ZXBL] H. Zhong, Q. Xie, M. T. Batchelor, C. Lee, Analytical eigenstates for the quantum Rabi model, J. Phys. A46, (2013), 415302; arXiv:1305.6782 [quant-ph]. 